\title{
Photometric and radial-velocity time-series of RR Lyrae stars in M3: analysis of single-mode variables
}

\author{
J. Jurcsik ${ }^{1 \star}$, P. Smitola ${ }^{1}$, G. Hajdu ${ }^{2,3}$, Á. Sódor ${ }^{1}$, J. Nuspl ${ }^{1}$, K. Kolenberg ${ }^{4,5}$, \\ G. Fürész ${ }^{8}$, L. G. Balázs ${ }^{1}$, C. Pilachowski ${ }^{9}$, A. Saha ${ }^{10}$, A. Moór ${ }^{1}$, E. Kun ${ }^{11,12}$, \\ A. Pál ${ }^{1}$, J. Bakos ${ }^{1}$, J. Kelemen ${ }^{1}$, T. Kovács ${ }^{13}$, L. Kriskovics ${ }^{1}$, K. Sárneczky ${ }^{1}$, \\ T. Szalai ${ }^{14}$, A. Szing ${ }^{15}$ K. Vida ${ }^{1}$ \\ ${ }^{1}$ Konkoly Observatory of the Hungarian Academy of Sciences, H-1121 Budapest, Konkoly Thege Miklós út 15-17., Hungary \\ ${ }^{2}$ Instituto de Astrofísica, Pontificia Universidad Católica de Chile, Av. Vicuña Mackenna 4860, 782-0436 Macul, Santiago, Chile \\ ${ }^{3}$ Instituto Milenio de Astrofísica, Santiago, Chile \\ ${ }^{4}$ Institute of Astronomy, KU Leuven, Celestijnenlaan 200D, 3001 Heverlee, Belgium \\ ${ }^{5}$ Physics Department, University of Antwerp, Groenenborgerlaan 171, B-2020 Antwerpen, Belgium \\ ${ }^{6}$ Institute of Astronomy, KU Leuven, Celestijnenlaan 200D, B-3001 Heverlee, Belgium \\ ${ }^{7}$ Harvard-Smithsonian Center for Astrophysics, 60 Garden Street, Cambridge MA 02138, USA \\ ${ }^{8}$ MIT Kavli Institute for Astrophysics and Space Research 77 Mass Ave 37-515, Cambridge, MA, 02139 \\ ${ }^{9}$ Department of Astronomy, Indiana University Bloomington, Swain West 319, 727 E. 3rd Street, Bloomington, IN 47405, US \\ ${ }^{10}$ National Optical Astronomy Observatories, Tucson, AZ 85726-6732, USA \\ ${ }^{11}$ Department of Theoretical Physics, University of Szeged, H-6720 Szeged, Tisza Lajos krt 84-86, Hungary \\ ${ }^{12}$ Department of Experimental Physics and Astronomical Observatory, University of Szeged, H-6720 Szeged, Dóm tér 9, Hungary \\ ${ }^{13}$ Institute of Theoretical Physics, Eotvos University, P.O. H-1518, Budapest, Hungary \\ ${ }^{14}$ Department of Optics and Quantum Electronics, University of Szeged, H-6720 Szeged, Dóm tér 9, Hungary \\ ${ }^{15}$ Baja Observatory, University of Szeged, 6500 Baja, KT: 766, Hungary
}

Accepted 2017 ..... Received 2017 ..., in original form

\begin{abstract}
We present the first simultaneous photometric and spectroscopic investigation of a large set of RR Lyrae variables in a globular cluster. The radial-velocity data presented comprise the largest sample of RVs of RR Lyrae stars ever obtained. The target is M3; $B V I_{\mathrm{C}}$ time-series of 111 and $b$ flux data of further 64 RRab stars, and RV data of 79 RR Lyrae stars are published. Blazhko modulation of the light curves of 47 percent of the RRab stars are detected. The mean value of the center-of-mass velocities of RR Lyrae stars is $-146.8 \mathrm{~km} \mathrm{~s}^{-1}$ with $4.52 \mathrm{~km} \mathrm{~s}^{-1}$ standard deviation, which is in good agreement with the results obtained for the red giants of the cluster. The $\Phi_{21} \mathrm{RV}$ phase difference of the RV curves of RRab stars is found to be uniformly constant both for the M3 and for Galactic field RRab stars; no period or metallicity dependence of the $\Phi_{21}{ }^{R V}$ is detected. The Baade-Wesselink distances of 26 non-Blazhko variables with the best phase-coverage radial-velocity curves are determined; the corresponding distance of the cluster, $10480 \pm 210 \mathrm{pc}$, agrees with the previous literature information. A quadratic formula for the $A_{\text {puls }}-A_{V}$ relation of RRab stars is given, which is valid for both OoI and OoII variables. We also show that the $(V-I)_{0}$ of RRab stars measured at light minimum is period dependent, there is at least 0.1 mag difference between the colours at minimum light of the shortest- and longest-period variables.
\end{abstract}

Key words: stars: horizontal branch - stars: oscillations (including pulsations) stars: variables: RR Lyrae - Galaxy: globular clusters: individual: M3 - techniques: photometric - techniques: spectroscopic
* E-mail: jurcsik@konkoly.hu

(C) 2016 The Authors

\section{INTRODUCTION}

Although the history of studying pulsating variables dates back more than a century, these investigations, using the 
most advanced-technique satellite and ground-based observations (e.g., HST, Kepler, Gaia, LSST, etc.), are still in the focus of modern astrophysics. Exploiting the advantage that globular clusters (GCs) are rich in RR Lyrae (RRL) type pulsating variable stars, and they provide relatively homogeneous samples of variables compared to other targets of massive photometric campaigns (e.g., Galactic field, Galactic bulge, satellite galaxies), GCs are frequent and popular targets of observations.

In spite of all these efforts, extended radial velocity (RV) surveys of RRL stars in GCs have not been undertaken. Complete RV curves were published for less than 50 Galactic field RRL stars and less than 10 RRLs in GCs previously. Photometric campaigns long and dense enough to reveal the modulation properties of RRL stars in GCs have not been performed previously, either. Because the origin of the lightcurve modulation, i.e. the Blazhko effect, which appears in about 50 percent of RRLs, is still unknown, a systematic study of Blazhko stars in GCs would provide important information on the Blazhko phenomenon. Therefore, the primary aim of our project was to collect extended multi-colour photometric data, as well as RV observations of the variables in one GC in order to perform a combined analysis of the data.

The GC, M3 (NGC5272) was selected as the target of the campaign, because it has one of the largest RRL population, and the variables are not too faint to observe spectroscopically. The large number of RRL stars in the cluster motivated many detailed investigations already. Without completeness, Cacciari et al. (2005) determined and analysed the physical properties of RRL stars (Cacciari et al. 2005), the light curves of the variables were modeled accurately using nonlinear pulsation models (Marconi \& Degl'Innocenti 2007), and the comparison of the observed properties of the variables and their distribution with canonical horizontal-branch models and evolutionary tracks led to important new conclusions (Marconi et al. 2003; Catelan 2004; Castellani et al. 2005). However, in spite of that M3 has already been the subject of several photometric [e.g., Kaluzny et al. (1998), Corwin \& Carney (2001), Clementini et al. (2004) Hartman et al. (2005), and Benkő et al. (2006)] and spectroscopic [e.g., Cohen \& Meléndez (2005), Johnson et al. (2005), Sneden et al. (2004) and Givens \& Pilachowski (2016)] studies, the available photometric data provide only uncertain and deficient information on the properties of the Blazhko stars of the cluster, and RV data of the variables has not been published previously.

Utilizing RV and photometric data, the BaadeWesselink (BW) method (as a review read Gautschy 1987) was long one of the most important tools to determine the distance of pulsating variables. Different variants of the method were successfully applied for dozens of Galactic field and some GC RRL stars (without completeness, e.g., Clementini et al. 1990; Cacciari et al. 1992; Carney et al. 1992; Fernley 1994; Liu \& Janes 1990a; Skillen et al. 1989, 1993; Storm et al. 1994b). Although, at the dawn of the Gaia era the determination of distances of clusters through the BW method may well become superseded, the method itself will continue to provide consistent distances of individual pulsating stars at large distances. We point out that for these stars (at distances comparable to M3 and farther away), the relative Gaia parallax error won't allow the derivation of better individual distances than those coming from the BW method. Moreover, the combined, parallel RV and photometric observations may still provide new information on the pulsation of stars.

In this paper, we present new, extended, multicolour photometric time-series of the fundamental mode RRL stars of M3 and also RV observations of the variables for the first time. The published RV data comprise the largest sample for RRL stars ever obtained. A detailed analysis of the photometric data of the overtone and double-mode variables, based on the same observational campaign, was already published in Jurcsik et al. (2015). The BW analysis of the singlemode variables of M3 is in the focus of the present paper; the study of Blazhko stars will be published separately.

\section{OBSERVATIONS AND DATA REDUCTION}

\subsection{Photometry}

Photometric time-series were collected in $B V I_{\mathrm{C}}$ bands with the 60/90 Schmidt telescope at Konkoly Observatory in 2012. Both aperture (IRAF/DAOPHOT/PHOT ${ }^{1}$ ) and Image Subtraction Method (ISIS, Alard 2000) photometry of the variables were performed. The light curves were transformed to the standard $B V I_{\mathrm{C}}$ system using 23 bright, photometrically stable standard stars, covering a wide range in colour, from the list of Stetson (2000). Full details of the observations, reduction and the photometry are given in Jurcsik et al. (2015), where the analysis of the overtone and the double-mode variables of M3, based on the data from the same photometric campaign, were published.

Standard $B V I_{\mathrm{C}}$ photometric time-series data of 111 fundamental-mode variables were obtained. Because of crowding, the magnitude calibration of the flux data derived from ISIS failed in the case of 64 RRab stars; instrumental $B$-band flux time series $(b)$ are given for these stars. The $B V I_{\mathrm{C}}$ magnitudes and the $b$ flux time-series of the fundamental-mode variables of M3 are available as Supplementary Material in the electronic edition of this article; Table 1 and Table 2 give samples of their form and content.

\subsection{Spectroscopy}

\subsubsection{Hectochelle@MMT measurements}

Parallel to the photometric measurements, spectroscopic data of M3 RRL stars were secured using the Hectochelle spectrograph attached to the 6.5-m MMT telescope in Arizona, USA (Szentgyörgyi et al. 2011) on 2 nights in March (JD2455991, 2455992) and on 3 nights in April (JD2 456023,2456025 and JD2 456 026), 2012.

Hectochelle is a multi-object echelle spectrograph capable of observing a single echelle-order of up to 240 point sources simultaneously with $R \approx 32000$ spectral resolution. The measurements covered the 5150 - $5300 \AA$ wavelength

1 IRAF is distributed by the National Optical Astronomy Observatories, which are operated by the Association of Universities for Research in Astronomy, Inc., under cooperative agreement with the National Science Foundation. 
Table 1. $B, V, I_{\mathrm{C}}$ CCD observations of $\mathrm{M} 3$ variables published in this paper. The full table is available as Supplementary Material in the online version of the journal.

\begin{tabular}{lccc}
\hline Star $^{a}$ & HJD-2 $400000^{b}$ & Mag & Band \\
\hline V001 & 55937.67595 & 16.373 & B \\
V001 & 55937.69025 & 16.321 & B \\
V001 & 55937.70074 & 16.367 & B \\
$\cdots$ & $\ldots$ & $\cdots$ & $\cdots$ \\
\hline
\end{tabular}

${ }^{a}$ Identification according to the catalogue of Samus et al. (2009).

${ }^{b}$ The HJDs represent the times of mid-exposure.

Table 2. Instrumental B-band flux time series (b) data of M3 variables with uncertain magnitudes on the reference frame. The complete table is given in the electronic edition.

\begin{tabular}{lcr}
\hline Star $^{a}$ & HJD-2 $400000^{b}$ & Flux $^{c}$ \\
\hline V004a & 55935.64720 & 9768.14 \\
V004a & 55937.67595 & -11001.05 \\
V004a & 55937.69025 & -8166.03 \\
$\ldots$ & $\ldots$ & $\ldots$ \\
\hline
\end{tabular}

${ }^{a}$ Identification according to the catalogue of Samus et al. (2009).

${ }^{b}$ The HJDs represent the times of mid-exposure.

${ }^{c}$ Flux differences derived from ISIS photometry (Alard 2000).

Table 3. Radial-velocity time-series data of M3 variables. The complete table is given in the electronic edition.

\begin{tabular}{lccc}
\hline Star & ${\text { HJD }-2400000^{a}}$ & RV $\left[\mathrm{km} \mathrm{s}^{-1}\right]$ & Tel./Instr. $^{b}$ \\
\hline V009 & 51600.791 & -185.93 & 1 \\
V009 & 51600.805 & -179.69 & 1 \\
V009 & 51600.818 & -190.43 & 1 \\
$\cdots$ & $\cdots$ & $\cdots$ & $\cdots$ \\
\hline
\end{tabular}

${ }^{a}$ The HJDs represent the times of mid-exposure.

b 1:Hydra@WIYN; 2:Hectochelle@MMT.

range containing the strong $\mathrm{Mg}$ triplet lines. Besides the target stars, several non-variable stars were also observed for calibration purposes.

The 20-min exposure CCD frames were bias and flatfield corrected, filtered for cosmics, then apertures were extracted and wavelength-calibrated using standard IRAF procedures by the instrument's pipeline. The spectra were then normalised and corrected for the barycentric motion. Finally, we calculated the cross-correlation function ( $\mathrm{CCF}$ ) of the calibrated spectra with a synthetic template F-star spectrum in the range of $[-250,-50] \mathrm{km} \mathrm{s}^{-1}$, and the RVs were determined by fitting a Gaussian function to the global maximum of the CCF. We checked for systematic uncertainties present in the deduced RV data by calculating the CCFs and RVs in several different ways; applying different templates ( $T_{\text {eff }}: 6500 / 7000 / 7250$ ), and selecting different sub-sets of spectral lines. It was found that these changes affected primarily the derived amplitude of the RV curve; hotter temperature and/or fewer lines gave rise to larger amplitudes. Nevertheless, the differences between the derived RV ampli- tudes do not exceed 5 percent of the total amplitude, i.e. it is smaller than $3 \mathrm{~km} \mathrm{~s}^{-1}$ even for the variables with the largest amplitude.

Due to the time consuming-robotic focal-plane fibreentrance manipulations of Hectochelle, ThAr wavelength reference spectra were obtained only before the first observing night. The RV stability was therefore ensured by relying on the RVs of the reference stars (not standards). However, because the RVs of only 4 reference stars proved to remain stable enough, the RV curves of stable-light-curve RRL stars were also used for RV calibration purposes, and the following co-trending method was applied. The raw pulsation RV curves of all the stable-light-curve RRab stars were pre-whitened by carrying out a preliminary Fourier-fit using pulsation periods determined from photometry. Then, the residuals of each exposure of a subsample of stars of adjacent fibre apertures, selected among the 4 reference stars and the stable-light-curve RRLs, were averaged. Finally, these averaged residuals were subtracted from each target's RV data in the corresponding aperture bin. These corrections were less than $\pm 1 \mathrm{~km} \mathrm{~s}^{-1}$ for the first run of the spectroscopy and were in the $2-4 \mathrm{~km} \mathrm{~s}^{-1}$ range for the second run. The 1- $\sigma$ stability of the reference stars and the rms scatter of appropriate order Fourier fits of stable-light-curve RRLs for the corrected RV data is better than $1 \mathrm{~km} \mathrm{~s}^{-1}$.

\subsubsection{Hydra@WIYN measurements}

RVs of RRL stars in M3 were also gathered using the Hydra fibre-feed spectrograph attached to the 3.5-m WIYN telescope at Kitt Peak on 5 nights (between JD 2451600 and 2451646 ) in 2000. The Hydra configurations included both RRL variables and red-giant-branch stars to provide velocity calibration. The observations and basic spectrum reductions were described in detail by Sandstrom et al. (2001). In brief, the Hydra observations were obtained at a resolving power of $R=3000$ and centered at a wavelength of $5100 \AA$.

$\mathrm{RVs}$ for all the variables and giants were derived using the IRAF task fxcor. Velocities were determined by crosscorrelation relative to the twilight-motr sky spectrum observed with the same instrument. The spectral region for cross-correlation was restricted to $5000-5400 \AA$ to avoid $\mathrm{H} \beta$. For the giants, the Tonry \& Davis (1979) "R" value was typically above 15 , while the RRL variables, with weaker lines and lower signal-to-noise ratio spectra, had values in the range $5<\mathrm{R}<20$. For the (non-variable) giants, the observed velocity uncertainties are typically $1 \mathrm{~km} \mathrm{~s}^{-1}$ based on the reproducibility of the measurements from observation to observation. The uncertainties of the RVs of the variables are estimated from the scatter about the Fourier fit of the RV curves of stable light-curve RRLs; the rms of these RV curves are $5-7 \mathrm{~km} \mathrm{~s}^{-1}$.

In each multi-object Hydra frame, the velocities of the giants were averaged to produce a mean giant velocity to establish the absolute calibration for RV. An offset was added to the measured RRL velocities in that frame to bring the velocities to the same systemic velocity defined by the giant stars. With a relatively small number of giants (only 14) to establish the cluster systemic velocity, our results are subject to sampling errors. These errors are estimated by considering randomly selected samples of 14 giants from the Soderberg et al. (1999) sample of 88 giants. The standard 

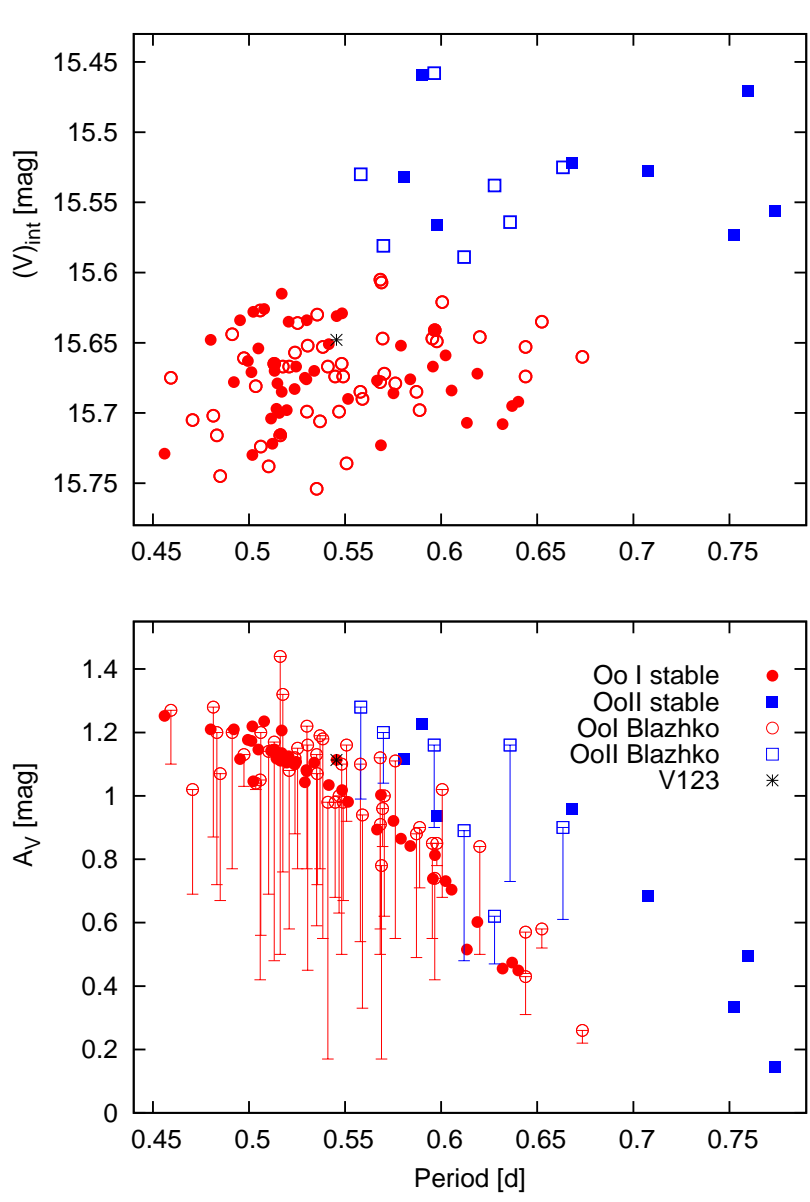

Figure 1. Intensity-averaged mean $V$ magnitude and peak to peak $V$ amplitude vs. pulsation period of RRab stars in M3 are plotted. Blazhko stars are shown at the largest amplitude, and their amplitude ranges are indicated by vertical bars. Beside the 2012 data, all the published CCD observations of Blazhko stars are checked in determining minimum/maximum values of their amplitudes.

deviation of the mean velocity of the sub-samples compared to the mean of the full sample of 88 giants is $0.8 \mathrm{~km} \mathrm{~s}^{-1}$. We take this as an estimate of the uncertainty of the RV zeropoint due to sampling error introduced by our small sample of giants.

\subsubsection{The RV data}

There are 16 non-Blazhko, single-mode, RRL stars with good pulsation-phase coverage in both RV data sets. The differences between the mean RVs ( $\gamma$-velocities) of these stars measured by the Hydra@WIYN and the Hectochelle@MMT instruments are in the $[-1.5,2.8] \mathrm{km} \mathrm{s}^{-1}$ range, with 0.6 mean value and $1.5 \mathrm{rms}$ scatter, indicating that there is no systematic difference between the two data sets exceeding the $\sim 1 \mathrm{~km} \mathrm{~s}^{-1}$ uncertainty.

Altogether 60 and $60-80$ RV measurements of 62 and 44 RRL stars were obtained by the Hectochelle@MMT and Hydra@WIYN instruments, respectively. Both data sets are available as Supplementary Material in the electronic edition of this article in a merged form. Table 3 supplies a sample for this data file showing its structure and the meaning of the columns.

\section{BASIC DATA OF THE VARIABLES}

The light and RV curves were analysed using the program packages MUFRAN (Kolláth 1990) and LCfit (Sódor 2012).

\subsection{Photometric results}

The length and the time-coverage of the photometric data made it possible to determine the light-curve solutions for most of the stars including the Blazhko-modulated ones. We have found that the light curve of about half ( 47 percent) of the measured RRab stars show the Blazhko effect. The true percentage of Blazhko stars may be even larger, as the photometry of the most crowded stars is too noisy to detect possible modulation.

The basic photometric results of stable-light-curve and Blazhko RRab stars are summarised in Table 4. The ID, the Oosterhoff type (based on the mean magnitudes and amplitudes as documented in Fig. 1), the pulsation period, the magnitude and intensity averaged $B V I_{\mathrm{C}}$ mean magnitudes, and the amplitudes - the smallest and largest amplitudes according to our observations for Blazhko stars - are listed. If larger or smaller $V$ amplitude of a Blazhko star has been detected in any of the published CCD data [Kaluzny et al. (1998), Corwin \& Carney (2001), Hartman et al. (2005), Benkő et al. (2006), and Jurcsik et al. (2012)], this extreme value of the amplitude is given in parenthesis.

Comparing the sample of the OoII stars with the results of Cacciari et al. (2005) a good agreement is found. Each of the stable-light-curve RRab stars classified as over-luminous by Cacciari et al. (2005) are OoII-type variable according to our photometry and vice versa. The Oosterhoff classification of some Blazhko stars (V035, V048, V067, V078 and V143) are, however, different, probably because the time coverage of the Corwin \& Carney (2001) observations does not make it possible to determine the mean magnitudes accurately enough if the amplitude of the light curve varies.

A more detailed analysis and comparison of the photometric and spectroscopic results of Blazhko and stable RRab stars is planned to be published in a separate paper.

\subsection{Radial-velocity results}

The RV data of overtone, non-Blazhko, and Blazhko RRab stars phased with the pulsation period are shown in Figs. 2, 3, and 4, respectively. Both the Hectochelle@MMT and the Hydra@WIYN data are plotted. The Hectochelle@MMT RV data cover fully or partially the pulsation cycles at two epochs separated by about 30 days. The RVs of these two observation runs are shown by different symbols for Blazhko stars.

The $\gamma$-velocities and RV amplitudes $\left(A_{\mathrm{RV}}\right)$ of overtone, single-mode and Blazhko RRab stars are listed in Table 5, 6 , and 7 , respectively. The 2 nd and 3 rd columns of these tables identify the RV observations of the star. Additional features of the light variations of the overtone variables (see 
Table 4. Summary of the photometric properties of RRab stars and the new overtone variable in M3.

\begin{tabular}{|c|c|c|c|c|c|c|c|c|c|c|c|c|c|c|}
\hline Star & $\begin{array}{c}\text { Period } \\
\mathrm{d}\end{array}$ & $\begin{array}{c}<B>^{a} \\
\text { mag }\end{array}$ & $\begin{array}{l}(B)^{a} \\
\text { mag }\end{array}$ & mag & $\begin{array}{l}A_{B}^{b} \\
\text { mag }\end{array}$ & $\begin{array}{c}<V> \\
\text { mag }\end{array}$ & $\begin{array}{l}(V) \\
\text { mag }\end{array}$ & $A_{V}^{b, c}$ & mag & $\begin{array}{c}<I_{\mathrm{C}}> \\
\text { mag }\end{array}$ & $\begin{array}{l}\left(I_{\mathrm{C}}\right) \\
\text { mag }\end{array}$ & mag & $\begin{array}{l}A_{I_{\mathrm{C}}}{ }^{b} \\
\text { mag }\end{array}$ & Comment \\
\hline V001 OoI & 0.52059 & 16.022 & 15.916 & 1.452 & & 15.694 & 15.635 & 1.125 & & 15.229 & 15.208 & 0.750 & & \\
\hline V003 OoII & 0.55820 & 15.908 & 15.803 & 1.62 & 1.36 & 15.588 & 15.530 & 1.28 & 0.99 & 15.129 & 15.106 & 0.85 & 0.68 & $\mathrm{Bl}$ \\
\hline V004a & 0.58504 & & & & & & & & & & & & & \\
\hline V004b & 0.59305 & & & & & & & & & & & & & $\mathrm{Bl}$ \\
\hline V005 OoI & 0.50579 & 15.974 & 15.932 & 1.38 & 0.60 & 15.650 & 15.627 & 1.05 & 0.42 & 15.202 & 15.193 & 0.65 & 0.28 & $\mathrm{Bl}$ \\
\hline V006 OoI & 0.51434 & 16.088 & 15.990 & 1.421 & & 15.751 & 15.697 & 1.118 & & 15.284 & 15.263 & 0.744 & & \\
\hline V007 OoI & 0.49742 & 16.052 & 15.943 & 1.50 & 1.38 & 15.718 & 15.661 & 1.13 & 1.03 & 15.260 & 15.238 & 0.77 & 0.65 & $\mathrm{Bl}$ \\
\hline V008 & 0.63671 & & & & & & & & & & & & & $\mathrm{Bl}$ \\
\hline V009 OoI & 0.54155 & 16.045 & 15.959 & 1.334 & & 15.697 & 15.651 & 1.034 & & 15.210 & 15.192 & 0.690 & & \\
\hline V010 OoI & 0.56955 & 16.042 & 15.981 & 1.23 & 1.06 & 15.680 & 15.647 & 0.96 & 0.84 & 15.169 & 15.157 & 0.64 & 0.53 & $\mathrm{Bl}$ \\
\hline V011 OoI & 0.50789 & 15.990 & 15.872 & 1.559 & & 15.694 & 15.626 & 1.235 & & 15.236 & 15.210 & 0.821 & & \\
\hline V014 OoII & 0.63590 & 15.960 & 15.892 & 1.46 & 0.97 & 15.602 & 15.564 & 1.16 & 0.73 & 15.107 & 15.092 & 0.76 & 0.50 & $\mathrm{Bl}$ \\
\hline V015 OoI & 0.53009 & 16.021 & 15.930 & 1.382 & & 15.684 & 15.634 & 1.078 & & 15.202 & 15.183 & 0.730 & & \\
\hline V016 OoI & 0.51149 & 16.100 & 15.998 & 1.462 & & 15.760 & 15.704 & 1.142 & & 15.288 & 15.266 & 0.761 & & \\
\hline V017 OoI & 0.57613 & 16.058 & 16.016 & 1.15 & 0.72 & 15.702 & 15.679 & $0.86(1.11)$ & 0.55 & 15.194 & 15.185 & 0.57 & 0.30 & $\mathrm{Bl}$ \\
\hline V018 OoI & 0.51645 & 16.106 & 16.011 & 1.421 & & 15.767 & 15.715 & 1.111 & & 15.295 & 15.274 & 0.749 & & \\
\hline V019 OoI & 0.63198 & 16.122 & 16.103 & 0.598 & & 15.718 & 15.708 & 0.455 & & 15.168 & 15.164 & 0.302 & & \\
\hline V020 OoI & 0.49126 & 15.998 & 15.916 & 1.53 & 1.00 & 15.691 & 15.644 & 1.20 & 0.77 & 15.248 & 15.230 & 0.76 & 0.45 & $\mathrm{Bl}$ \\
\hline V021 OoI & 0.51577 & 16.103 & 16.007 & 1.437 & & 15.753 & 15.700 & 1.115 & & 15.278 & 15.258 & 0.741 & & \\
\hline V022 OoI & 0.48143 & 16.042 & 15.948 & 1.60 & 1.15 & 15.757 & 15.702 & 1.28 & 0.87 & 15.337 & 15.316 & 0.86 & 0.55 & $\mathrm{Bl}$ \\
\hline V023 OoI & 0.59538 & 16.012 & 15.972 & 1.10 & 0.70 & 15.669 & 15.647 & 0.85 & 0.55 & 15.137 & 15.129 & 0.56 & 0.36 & $\mathrm{Bl}$ \\
\hline V024 OoII & 0.66343 & 15.953 & 15.908 & 1.18 & 0.78 & 15.549 & 15.525 & 0.90 & 0.61 & & & & & $\mathrm{Bl}$ \\
\hline V025 OoI & 0.48007 & 16.054 & 15.932 & 1.550 & & 15.714 & 15.648 & 1.210 & & 15.271 & 15.244 & 0.807 & & \\
\hline V026 OoII & 0.59774 & 15.959 & 15.892 & 1.208 & & 15.602 & 15.566 & 0.937 & & 15.093 & 15.079 & 0.621 & & \\
\hline V027 OoI & 0.57906 & 16.048 & 15.994 & 1.116 & & 15.682 & 15.652 & 0.865 & & 15.200 & 15.188 & 0.576 & & \\
\hline V028 OoI & 0.47066 & 15.978 & 15.921 & 1.28 & 1.12 & 15.741 & 15.705 & 1.02 & $0.88(0.69)$ & 15.312 & 15.298 & 0.66 & 0.55 & $\mathrm{Bl}$ \\
\hline V030 & 0.51209 & & & & & & & & & & & & & \\
\hline V031 OoII & 0.58072 & 15.910 & 15.815 & 1.445 & & 15.584 & 15.532 & 1.118 & & 15.145 & 15.123 & 0.766 & & \\
\hline V032 OoI & 0.49535 & 16.030 & 15.924 & 1.455 & & 15.691 & 15.634 & 1.116 & & 15.276 & 15.253 & 0.754 & & \\
\hline V033 OoI & 0.52524 & 15.996 & 15.926 & 1.50 & 1.06 & 15.674 & 15.636 & 1.15 & 0.77 & 15.190 & 15.176 & 0.77 & 0.46 & $\mathrm{Bl}$ \\
\hline V034 OoI & 0.55901 & 16.061 & 16.014 & 1.25 & 0.50 & 15.714 & 15.690 & 0.94 & 0.33 & 15.219 & 15.209 & 0.65 & 0.19 & $\mathrm{Bl}$ \\
\hline V035 OoI & 0.53058 & 16.010 & 15.958 & 1.53 & 0.58 & 15.681 & 15.652 & 1.16 & 0.45 & 15.204 & 15.193 & 0.74 & 0.26 & $\mathrm{Bl}$ \\
\hline V036 OoI & 0.54560 & 16.048 & 15.950 & 1.457 & & 15.684 & 15.631 & 1.114 & & 15.212 & 15.190 & 0.755 & & \\
\hline V038 OoI & 0.55801 & 16.062 & 16.008 & 1.50 & 0.71 & 15.715 & 15.685 & 1.10 & 0.54 & 15.215 & 15.204 & 0.70 & 0.37 & $\mathrm{Bl}$ \\
\hline V039 OoI & 0.58710 & 16.066 & 16.030 & 1.13 & 0.70 & 15.704 & 15.685 & 0.88 & 0.49 & 15.190 & 15.182 & 0.57 & 0.30 & $\mathrm{Bl}$ \\
\hline V040 OoI & 0.55154 & 16.088 & 16.015 & 1.274 & & 15.730 & 15.690 & 0.981 & & 15.234 & 15.219 & 0.658 & & \\
\hline V041 OoI & 0.48504 & 16.078 & 15.995 & 1.33 & 1.17 & 15.793 & 15.745 & 1.07 & $0.90(0.67)$ & 15.356 & 15.338 & 0.70 & 0.57 & $\mathrm{Bl}$ \\
\hline V042 OoII & 0.59007 & 15.869 & 15.749 & 1.609 & & 15.524 & 15.459 & 1.226 & & 15.063 & 15.037 & 0.816 & & \\
\hline V043 & 0.54053 & & & & & & & & & & & & & $\mathrm{Bl}$ \\
\hline V045 OoI & 0.53691 & 16.092 & 16.021 & 1.54 & 1.00 & 15.745 & 15.706 & 1.19 & 0.77 & 15.260 & 15.245 & 0.80 & 0.46 & $\mathrm{Bl}$ \\
\hline V046 OoI & 0.61338 & 16.109 & 16.086 & 0.692 & & 15.719 & 15.707 & 0.515 & & 15.173 & 15.168 & 0.345 & & \\
\hline V047 OoI & 0.54107 & 16.000 & 15.967 & 1.25 & 0.20 & 15.684 & 15.667 & 0.98 & 0.17 & 15.207 & 15.201 & 0.65 & 0.13 & $\mathrm{Bl}$ \\
\hline V048 OoI & 0.62783 & 15.942 & 15.918 & 0.80 & 0.60 & 15.551 & 15.538 & 0.62 & 0.47 & 14.994 & 14.989 & 0.40 & 0.33 & $\mathrm{Bl}$ \\
\hline V049 OoI & 0.54821 & 16.058 & 15.977 & 1.48 & 1.17 & 15.710 & 15.665 & 1.10 & $0.90(0.50)$ & 15.233 & 15.215 & 0.75 & 0.58 & $\mathrm{Bl}$ \\
\hline V050 OoI & 0.51309 & 16.041 & 15.954 & 1.53 & 1.04 & 15.713 & 15.665 & 1.17 & $0.78(0.48)$ & 15.233 & 15.215 & 0.78 & 0.53 & $\mathrm{Bl}$ \\
\hline V051 OoI & 0.58397 & 16.090 & 16.036 & 1.105 & & 15.705 & 15.676 & 0.842 & & 15.192 & 15.180 & 0.576 & & \\
\hline V052 OoI & 0.51624 & 16.096 & 16.015 & 1.88 & 0.95 & 15.761 & 15.716 & 1.44 & $0.69(0.50)$ & 15.285 & 15.267 & 0.96 & 0.46 & $\mathrm{Bl}$ \\
\hline V053 OoI & 0.50488 & 16.074 & 15.961 & 1.530 & & 15.713 & 15.654 & 1.146 & & 15.263 & 15.239 & 0.798 & & \\
\hline V054 OoI & 0.50611 & 16.072 & 16.032 & 1.57 & 0.78 & 15.748 & 15.724 & 1.20 & 0.56 & 15.310 & 15.300 & 0.82 & 0.27 & $\mathrm{Bl}$ \\
\hline V055 OoI & 0.52983 & 16.064 & 15.972 & 1.380 & & 15.727 & 15.676 & 1.081 & & 15.232 & 15.213 & 0.718 & & \\
\hline V057 OoI & 0.51218 & 16.101 & 16.001 & 1.435 & & 15.779 & 15.722 & 1.145 & & 15.318 & 15.296 & 0.758 & & \\
\hline V058 OoI & 0.51705 & 16.026 & 15.902 & 1.594 & & 15.681 & 15.615 & 1.206 & & 15.231 & 15.205 & 0.803 & & \\
\hline V059 OoI & 0.58882 & 16.095 & 16.045 & 1.16 & 0.90 & 15.725 & 15.698 & 0.90 & 0.71 & 15.204 & 15.194 & 0.59 & 0.40 & $\mathrm{Bl}$ \\
\hline V060 OoII & 0.70773 & 15.947 & 15.909 & 0.922 & & 15.547 & 15.528 & 0.683 & & 15.023 & 15.015 & 0.448 & & \\
\hline V061* OoI & 0.52091 & 16.021 & 15.958 & 1.44 & 0.77 & 15.702 & 15.667 & 1.08 & 0.58 & 15.231 & 15.217 & 0.67 & 0.36 & $\mathrm{Bl} / \mathrm{dm}$ \\
\hline V062 OoI & 0.65240 & 16.046 & 16.022 & 0.77 & 0.69 & 15.648 & 15.635 & 0.58 & 0.52 & 15.097 & 15.092 & 0.37 & 0.33 & $\mathrm{Bl}$ \\
\hline V063 OoI & 0.57040 & 16.054 & 16.002 & 1.30 & 0.82 & 15.701 & 15.672 & 1.00 & 0.62 & 15.188 & 15.178 & 0.66 & 0.38 & $\mathrm{Bl}$ \\
\hline V064 OoI & 0.60546 & 16.084 & 16.045 & 0.942 & & 15.705 & 15.684 & 0.704 & & 15.165 & 15.157 & 0.472 & & \\
\hline V065 OoII & 0.66835 & 15.922 & 15.856 & 1.229 & & 15.559 & 15.522 & 0.959 & & 15.041 & 15.026 & 0.629 & & \\
\hline V066 OoI & 0.62015 & 16.048 & 16.016 & 1.06 & 0.63 & 15.662 & 15.646 & 0.84 & 0.50 & 15.121 & 15.115 & 0.52 & 0.30 & $\mathrm{Bl}$ \\
\hline V067 OoI & 0.56832 & 16.062 & 16.012 & 1.47 & 0.66 & 15.705 & 15.678 & 1.12 & 0.50 & 15.195 & 15.184 & 0.75 & 0.32 & $\mathrm{Bl}$ \\
\hline V069 OoI & 0.56662 & 16.085 & 16.025 & 1.187 & & 15.710 & 15.677 & 0.894 & & 15.196 & 15.184 & 0.600 & & \\
\hline
\end{tabular}


Table 4 - continued

\begin{tabular}{|c|c|c|c|c|c|c|c|c|c|c|c|c|c|c|}
\hline Star & $\begin{array}{c}\text { Period } \\
\mathrm{d}\end{array}$ & $\begin{array}{c}<B>^{a} \\
\operatorname{mag}\end{array}$ & $\begin{array}{l}(B)^{a} \\
\text { mag }\end{array}$ & $\operatorname{mag}^{A}$ & $\begin{array}{l}A_{B}^{b} \\
\text { mag }\end{array}$ & $\begin{array}{c}<V> \\
\operatorname{mag}\end{array}$ & $\begin{array}{l}(V) \\
\text { mag }\end{array}$ & mag & $\begin{array}{c}A_{V}^{b, c} \\
\text { mag }\end{array}$ & $\begin{array}{c}<I_{\mathrm{C}}> \\
\operatorname{mag}\end{array}$ & $\begin{array}{l}\left(I_{\mathrm{C}}\right) \\
\mathrm{mag}\end{array}$ & mag & $\begin{array}{l}A_{I_{\mathrm{C}}}{ }^{b} \\
\mathrm{mag}\end{array}$ & Comment \\
\hline V071 OoI & 0.54905 & 16.054 & 15.999 & 1.25 & 0.80 & 15.706 & 15.674 & 0.98 & 0.67 & 15.206 & 15.195 & 0.63 & 0.43 & $\mathrm{Bl}$ \\
\hline V072 OoI & 0.45608 & 16.115 & 15.982 & 1.611 & & 15.803 & 15.729 & 1.252 & & 15.378 & 15.349 & 0.841 & & \\
\hline V073 OoI & 0.67349 & 16.065 & 16.059 & 0.36 & 0.28 & 15.663 & 15.660 & 0.26 & 0.22 & 15.104 & 15.103 & 0.16 & 0.14 & $\mathrm{Bl}$ \\
\hline V074 OoI & 0.49215 & 16.097 & 15.976 & 1.565 & & 15.744 & 15.678 & 1.210 & & 15.315 & 15.288 & 0.849 & & \\
\hline V076 OoI & 0.50176 & 16.105 & 15.986 & 1.568 & & 15.797 & 15.730 & 1.219 & & 15.335 & 15.308 & 0.823 & & \\
\hline V077 OoI & 0.45935 & 16.057 & 15.931 & 1.70 & 1.50 & 15.742 & 15.675 & 1.27 & 1.10 & 15.365 & 15.337 & 0.90 & 0.76 & $\mathrm{Bl}$ \\
\hline V078 OoII? & 0.61192 & 15.985 & 15.947 & 1.21 & 0.68 & 15.609 & 15.589 & 0.89 & 0.48 & 15.059 & 15.051 & 0.61 & 0.33 & $\mathrm{Bl}$ \\
\hline V079 OoI & 0.48329 & 16.065 & 15.998 & 1.58 & 0.93 & 15.755 & 15.716 & 1.20 & 0.72 & 15.306 & 15.291 & 0.77 & 0.49 & $\mathrm{Bl}$ \\
\hline V080 OoI & 0.5384 & 16.048 & 15.966 & 1.50 & 1.20 & 15.700 & 15.653 & 1.18 & $0.92(0.55)$ & 15.222 & 15.203 & 0.77 & 0.59 & $\mathrm{Bl}$ \\
\hline V081 OoI & 0.52912 & 16.057 & 15.971 & 1.357 & & 15.722 & 15.675 & 1.043 & & 15.235 & 15.217 & 0.695 & & \\
\hline V082 OoI & 0.52454 & 16.053 & 15.957 & 1.399 & & 15.721 & 15.667 & 1.107 & & 15.240 & 15.219 & 0.743 & & \\
\hline V083 OoI & 0.50127 & 16.061 & 15.951 & 1.494 & & 15.733 & 15.671 & 1.173 & & 15.276 & 15.252 & 0.793 & & \\
\hline V084 OoI & 0.59572 & 16.070 & 16.028 & 0.955 & & 15.689 & 15.667 & 0.739 & & 15.153 & 15.144 & 0.486 & & \\
\hline V089 OoI & 0.54848 & 16.029 & 15.951 & 1.313 & & 15.671 & 15.629 & 1.018 & & 15.185 & 15.169 & 0.661 & & \\
\hline V090 OoI & 0.51703 & 16.074 & 15.974 & 1.438 & & 15.741 & 15.685 & 1.135 & & 15.264 & 15.242 & 0.756 & & \\
\hline V091 OoI & 0.53013 & 16.072 & 15.988 & 1.38 & 1.03 & 15.752 & 15.699 & 1.22 & 0.77 & 15.213 & 15.197 & 0.75 & 0.46 & $\mathrm{Bl}$ \\
\hline V092 OoI & 0.50355 & 16.055 & 15.966 & 1.33 & 1.32 & 15.731 & 15.681 & 1.04 & 1.02 & 15.265 & 15.247 & 0.66 & 0.64 & $\mathrm{Bl}$ \\
\hline V093 OoI & 0.60230 & 16.053 & 16.012 & 0.954 & & 15.681 & 15.659 & 0.731 & & 15.143 & 15.134 & 0.471 & & \\
\hline V094 OoI & 0.52370 & 16.075 & 15.981 & 1.398 & & 15.735 & 15.683 & 1.099 & & 15.257 & 15.236 & 0.739 & & \\
\hline V096 OoI & 0.49941 & 16.038 & 15.925 & 1.509 & & 15.726 & 15.663 & 1.177 & & 15.248 & 15.224 & 0.769 & & \\
\hline V100 OoI & 0.61882 & 16.082 & 16.052 & 0.809 & & 15.687 & 15.672 & 0.602 & & 15.173 & 15.166 & 0.415 & & \\
\hline V101 OoI & 0.64389 & 16.099 & 16.076 & 0.79 & 0.58 & 15.686 & 15.674 & 0.57 & 0.44 & 15.136 & 15.131 & 0.38 & 0.28 & $\mathrm{Bl}$ \\
\hline V104 OoII & 0.56993 & 15.980 & 15.878 & 1.55 & 1.38 & 15.635 & 15.581 & 1.20 & 1.04 & 15.162 & 15.141 & 0.80 & 0.69 & $\mathrm{Bl}$ \\
\hline V106 OoI & 0.54689 & 16.061 & 16.009 & 1.25 & 0.85 & 15.729 & 15.699 & 1.00 & 0.63 & 15.260 & 15.248 & 0.68 & 0.37 & $\mathrm{Bl}$ \\
\hline V108 OoI & 0.51961 & 16.086 & 15.991 & 1.408 & & 15.752 & 15.698 & 1.105 & & 15.275 & 15.255 & 0.750 & & \\
\hline V109 OoI & 0.53391 & 16.078 & 15.985 & 1.412 & & 15.721 & 15.670 & 1.105 & & 15.223 & 15.204 & 0.730 & & \\
\hline V110 OoI & 0.53547 & 16.010 & 15.942 & 1.43 & 0.94 & 15.665 & 15.630 & 1.07 & 0.72 & 15.206 & 15.192 & 0.73 & 0.48 & $\mathrm{Bl}$ \\
\hline V111 OoI & 0.51019 & 16.079 & 16.014 & 1.51 & 0.96 & 15.772 & 15.738 & 1.14 & 0.69 & 15.270 & 15.256 & 0.75 & 0.44 & $\mathrm{Bl}$ \\
\hline V113 OoI & 0.51301 & 16.055 & 15.952 & 1.460 & & 15.723 & 15.665 & 1.143 & & 15.260 & 15.237 & 0.772 & & \\
\hline V114 OoI & 0.59773 & 16.046 & 15.997 & 1.09 & 1.00 & 15.676 & 15.649 & 0.85 & 0.78 & 15.172 & 15.162 & 0.53 & 0.49 & $\mathrm{Bl}$ \\
\hline V115 OoI & 0.51335 & 16.055 & 15.953 & 1.448 & & 15.728 & 15.670 & 1.146 & & 15.247 & 15.225 & 0.764 & & \\
\hline V116 OoI & 0.51481 & 16.067 & 15.966 & 1.436 & & 15.734 & 15.679 & 1.120 & & 15.264 & 15.242 & 0.747 & & \\
\hline V117 OoI & 0.60054 & 16.005 & 15.950 & 1.32 & 0.90 & 15.652 & 15.621 & 1.02 & 0.68 & 15.137 & 15.126 & 0.67 & 0.42 & $\mathrm{Bl}$ \\
\hline V118** OoI & 0.49941 & & & & & & & & & & & & & \\
\hline V119 OoI & 0.51758 & 16.051 & 15.969 & 1.60 & 1.05 & 15.711 & 15.667 & 1.32 & 0.76 & 15.247 & 15.230 & 0.87 & 0.46 & $\mathrm{Bl}$ \\
\hline V120 OoI & 0.64014 & 16.088 & 16.072 & 0.572 & & 15.701 & 15.692 & 0.449 & & 15.137 & 15.134 & 0.302 & & \\
\hline V121 OoI & 0.53520 & 16.116 & 16.063 & 1.46 & 0.83 & 15.784 & 15.754 & 1.13 & 0.59 & 15.309 & 15.298 & 0.75 & 0.32 & $\mathrm{Bl}$ \\
\hline V122 & 0.49762 & & & & & & & & & & & & & \\
\hline V123 OoI & 0.54547 & 16.043 & 15.952 & 1.404 & & 15.700 & 15.648 & 1.113 & & 15.195 & 15.174 & 0.734 & & \\
\hline V124 OoII & 0.75243 & 15.987 & 15.977 & 0.449 & & 15.579 & 15.573 & 0.335 & & 15.004 & 15.001 & 0.233 & & \\
\hline V130 OoI & 0.56899 & 16.049 & 16.035 & 1.14 & 0.26 & 15.614 & 15.607 & 0.78 & 0.17 & 15.088 & 15.085 & 0.53 & 0.12 & $\mathrm{Bl}$ \\
\hline V133 OoI & 0.55073 & 16.107 & 16.032 & 1.45 & 1.10 & 15.779 & 15.736 & 1.16 & 0.92 & 15.221 & 15.208 & 0.64 & 0.54 & $\mathrm{Bl}$ \\
\hline V134 & 0.61805 & & & & & & & & & $\mathrm{Bl}$ & & & & \\
\hline V135 OoI & 0.56839 & 15.962 & 15.918 & 1.17 & 0.80 & 15.630 & 15.605 & 0.91 & 0.58 & 15.181 & 15.171 & 0.60 & 0.36 & $\mathrm{Bl}$ \\
\hline V136 & 0.61720 & & & & & & & & & & & & & \\
\hline V137 OoI & 0.57515 & 16.066 & 16.003 & 1.193 & & 15.720 & 15.686 & 0.921 & & 15.185 & 15.172 & 0.598 & & \\
\hline V139 & 0.55999 & & & & & & & & & & & & & \\
\hline V142 OoI & 0.56863 & 16.120 & 16.047 & 1.297 & & 15.763 & 15.723 & 1.003 & & 15.318 & 15.301 & 0.704 & & \\
\hline V143 OoII & 0.59641 & 15.846 & 15.772 & 1.54 & 1.20 & 15.495 & 15.458 & 1.16 & 0.90 & 15.035 & 15.016 & 0.78 & 0.60 & $\mathrm{Bl}$ \\
\hline V144*** OoI & 0.59670 & 16.043 & 16.024 & 0.60 & 0.60 & 15.649 & 15.641 & $0.42(0.74)$ & 0.42 & 15.115 & 15.112 & 0.25 & 0.25 & $\mathrm{Bl}$ \\
\hline V145 & 0.51449 & & & & & & & & & & & & & \\
\hline V146 OoI & 0.50219 & 16.022 & 15.926 & 1.402 & & 15.677 & 15.628 & 1.046 & & 15.254 & 15.235 & 0.708 & & \\
\hline V148 & 0.46728 & & & & & & & & & & & & & \\
\hline V149 & 0.54816 & & & & & & & & & & & & & $\mathrm{Bl}$ \\
\hline V150 OoI & 0.52392 & 16.038 & 15.954 & 1.50 & 1.10 & 15.700 & 15.657 & 1.12 & 0.88 & 15.220 & 15.205 & 0.72 & 0.54 & $\mathrm{Bl}$ \\
\hline V151 & 0.51682 & & & & & & & & & & & & & $\mathrm{Bl}$ \\
\hline V156 & 0.53200 & & & & & & & & & & & & & \\
\hline V157 & 0.54285 & & & & & & & & & & & & & $\mathrm{Bl}$ \\
\hline V159 & 0.53382 & & & & & & & & & & & & & $\mathrm{Bl}$ \\
\hline V160 & 0.65730 & & & & & & & & & & & & & $\mathrm{Bl}$ \\
\hline V161 & 0.52656 & & & & & & & & & & & & & $\mathrm{Bl}$ \\
\hline V165 & 0.48363 & & & & & & & & & & & & & $\mathrm{Bl}$ \\
\hline
\end{tabular}


Table 4 - continued

\begin{tabular}{|c|c|c|c|c|c|c|c|c|c|c|c|c|c|c|}
\hline Star & $\begin{array}{c}\text { Period } \\
\text { d }\end{array}$ & $\begin{array}{c}<B>^{a} \\
\operatorname{mag}\end{array}$ & $\begin{array}{l}(B)^{a} \\
\text { mag }\end{array}$ & $\operatorname{mag}{ }^{A}$ & $\begin{array}{l}A_{B}^{b} \\
\text { mag }\end{array}$ & $\begin{array}{c}<V> \\
\operatorname{mag}\end{array}$ & $\begin{array}{l}(V) \\
\text { mag }\end{array}$ & mag & $\begin{array}{l}A_{V}^{b, c} \\
\mathrm{mag}\end{array}$ & $\begin{array}{c}<I_{\mathrm{C}}> \\
\operatorname{mag}\end{array}$ & $\begin{array}{l}\left(I_{\mathrm{C}}\right) \\
\mathrm{mag}\end{array}$ & mag & $\begin{array}{l}A_{I_{\mathrm{C}}}{ }^{b} \\
\mathrm{mag}\end{array}$ & Comment \\
\hline V167 OoI & 0.64395 & 16.063 & 16.051 & 0.55 & 0.43 & 15.659 & 15.653 & 0.43 & 0.31 & 15.127 & 15.122 & 0.27 & 0.21 & $\mathrm{Bl}$ \\
\hline V172 & 0.54229 & & & & & & & & & & & & & \\
\hline V173 & 0.60700 & & & & & & & & & & & & & \\
\hline V174 & 0.59123 & & & & & & & & & & & & & $\mathrm{Bl}$ \\
\hline V175 & 0.56970 & & & & & & & & & & & & & \\
\hline V176 & 0.53959 & & & & & & & & & & & & & $\mathrm{Bl}$ \\
\hline V180 & 0.60910 & & & & & & & & & & & & & \\
\hline V181 & 0.66380 & & & & & & & & & & & & & \\
\hline V184 & 0.53128 & & & & & & & & & & & & & $\mathrm{Bl}$ \\
\hline V186 & 0.66327 & & & & & & & & & & & & & $\mathrm{Bl}$ \\
\hline V187 & 0.58620 & & & & & & & & & & & & & \\
\hline V189 & 0.61292 & & & & & & & & & & & & & \\
\hline V190 & 0.52280 & & & & & & & & & & & & & \\
\hline V191 & 0.51918 & & & & & & & & & & & & & $\mathrm{Bl}$ \\
\hline V192 & 0.49727 & & & & & & & & & & & & & $\mathrm{Bl}$ \\
\hline V193 & 0.74786 & & & & & & & & & & & & & \\
\hline V194 & 0.48920 & & & & & & & & & & & & & \\
\hline V195 & 0.64397 & & & & & & & & & & & & & $\mathrm{Bl}$ \\
\hline V197 & 0.49995 & & & & & & & & & & & & & \\
\hline V201 & 0.54052 & & & & & & & & & & & & & $\mathrm{Bl}$ \\
\hline V202 OoII & 0.77359 & 15.962 & 15.960 & 0.191 & & 15.557 & 15.556 & 0.146 & & 14.983 & 14.983 & 0.096 & & \\
\hline V205 OoI & 0.63691 & 16.105 & 16.085 & 0.627 & & 15.706 & 15.695 & 0.474 & & 15.154 & 15.149 & 0.322 & & \\
\hline V211 & 0.55820 & & & & & & & & & & & & & $\mathrm{Bl}$ \\
\hline V212 & 0.54222 & & & & & & & & & & & & & $\mathrm{Bl}$ \\
\hline V214 & 0.53953 & & & & & & & & & & & & & $\mathrm{Bl}$ \\
\hline V215 & 0.52863 & & & & & & & & & & & & & \\
\hline V218 OoI & 0.54487 & 16.075 & 16.013 & 1.29 & 0.90 & 15.706 & 15.674 & 0.98 & 0.68 & 15.203 & 15.190 & 0.66 & 0.40 & $\mathrm{Bl}$ \\
\hline V219 & 0.61363 & & & & & & & & & & & & & $\mathrm{Bl}$ \\
\hline V220 & 0.60016 & & & & & & & & & & & & & $\mathrm{Bl}$ \\
\hline V222 OoI & 0.59674 & 16.081 & 16.027 & 1.106 & & 15.668 & 15.641 & 0.813 & & 15.168 & 15.157 & 0.550 & & \\
\hline V226 & 0.48844 & & & & & & & & & & & & & \\
\hline V229 & 0.51608 & & & & & & & & & & & & & \\
\hline V234 & 0.50806 & & & & & & & & & & & & & \\
\hline V235 OoII & 0.75985 & 15.853 & 15.834 & 0.632 & & 15.482 & 15.471 & 0.495 & & 14.877 & 14.873 & 0.328 & & \\
\hline V239 & 0.50399 & & & & & & & & & & & & & $\mathrm{Bl}$ \\
\hline V241 & 0.59615 & & & & & & & & & & & & & \\
\hline V243 & 0.63462 & & & & & & & & & & & & & $\mathrm{Bl}$ \\
\hline V244 & 0.53785 & & & & & & & & & & & & & \\
\hline V247 & 0.60535 & & & & & & & & & & & & & \\
\hline V248 & 0.50980 & & & & & & & & & & & & & \\
\hline V249 & 0.53300 & & & & & & & & & & & & & \\
\hline V250a & 0.5744 & & & & & & & & & & & & & \\
\hline V250b & 0.5924 & & & & & & & & & & & & & \\
\hline V254 & 0.60563 & & & & & & & & & & & & & \\
\hline V255 & 0.57265 & & & & & & & & & & & & & $\mathrm{Bl}$ \\
\hline V257 & 0.60205 & & & & & & & & & & & & & \\
\hline V258 & 0.71340 & & & & & & & & & & & & & \\
\hline V262 & 0.65508 & & & & & & & & & & & & & \\
\hline V270a & 0.69018 & & & & & & & & & & & & & \\
\hline V270b & 0.62585 & & & & & & & & & & & & & $\mathrm{Bl}$ \\
\hline V271 & 0.63280 & & & & & & & & & & & & & \\
\hline \multicolumn{15}{|l|}{ New Var } \\
\hline N.V. & 0.24924 & 15.897 & 15.897 & 0.066 & & 15.675 & 15.675 & 0.051 & & 15.453 & 15.452 & 0.033 & & \\
\hline
\end{tabular}

$a<>$ and () denote magnitude and intensity averaged mean magnitudes, respectively.

${ }^{b}$ Peak to peak amplitudes; the observed largest- and smallest-amplitudes of Blazhko stars.

${ }^{c}$ Larger/smaller extreme value of the peak to peak amplitude according to other data is given in parenthesis.

* $f_{1}$ appears in the residual with 0.005 mag amplitude in the $V$ band $\left(f_{1}=2.5799 \mathrm{~d}^{-1} ; P_{1}=.3876 \mathrm{~d} ; P_{1} / P_{0}=0.744\right)$.

** Incomplete phase coverage, mean magnitudes and amplitudes cannot be determined.

*** Amplitude modulation on a very long time-scale, see fig. 2. in Jurcsik \& Smitola (2016). 
1st overtone

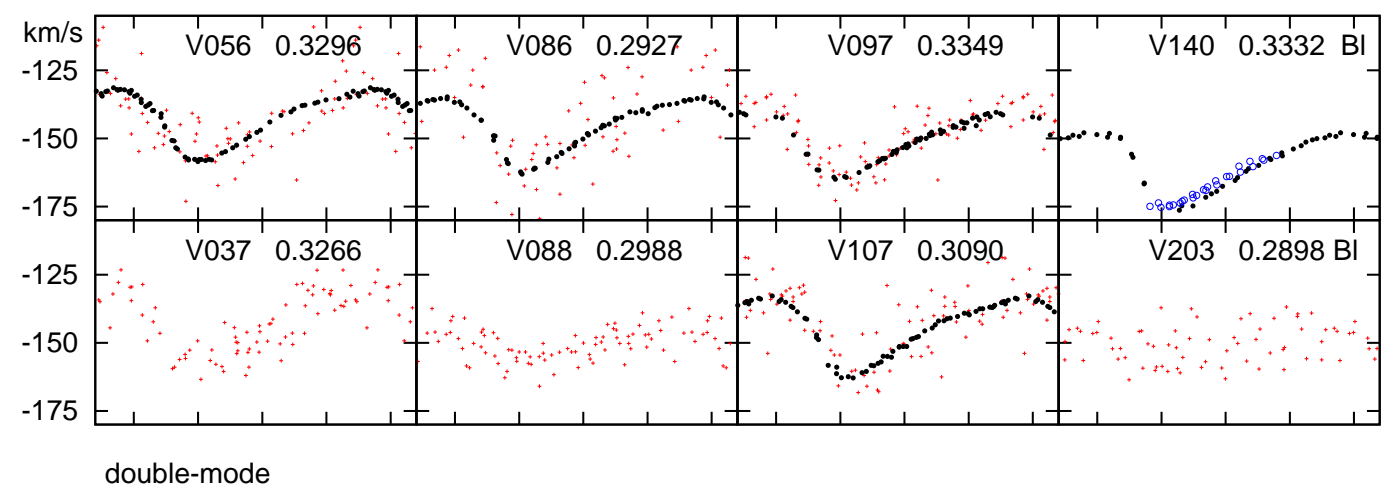

double-mode 2nd overtone

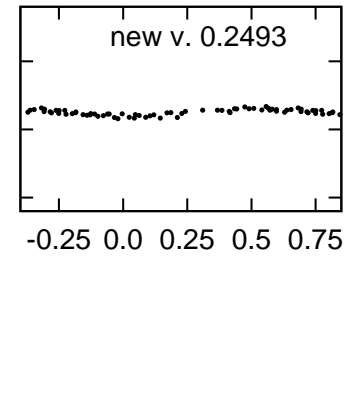

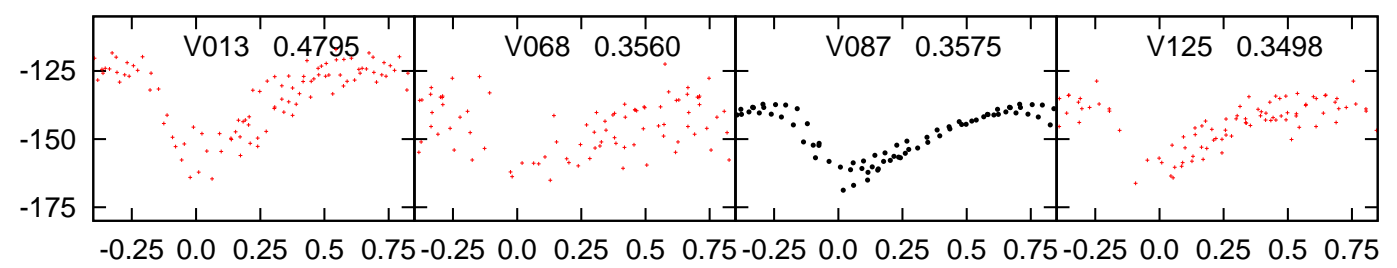

Figure 2. Radial-velocity curves of overtone and double-mode RR Lyrae stars in M3. The Hectoechelle@MMT and the Hydra@WIYN RV data are shown by black dots ans red crosses, respectively. The radial velocity data of the first (black dots) and second run (blue circles) of the Hectoechelle@MMT observations for the Blazhko star, V140, are shown by different symbols. The IDs and the pulsation period (days) of the variables are also given in the panels.

Table 5. Summary of the radial-velocity data of overtone and double-mode RR Lyrae stars in M3.

\begin{tabular}{lccccl}
\hline Star & \multicolumn{2}{c}{ Observation } & $\gamma$ & $A_{\mathrm{RV}}$ & Comment $^{a}$ \\
& Hydra & Hect. & {$\left[\mathrm{km} \mathrm{s}^{-1}\right]$} & {$\left[\mathrm{km} \mathrm{s}^{-1}\right]$} & \\
\hline V013 & $\mathrm{x}$ & - & -139.0 & 35.0 & $\mathrm{dm} f_{0.61}$ \\
V037 & $\mathrm{x}$ & - & -143.2 & 26.6 & $f_{0.61}$ \\
V056 & $\mathrm{x}$ & $\mathrm{x}$ & -143.4 & 26.2 & $f_{0.61}$ \\
V068 & $\mathrm{x}$ & - & -148.7 & 23.5 & $\mathrm{dm} f_{0.61}$ \\
V086 & $\mathrm{x}$ & $\mathrm{x}$ & -146.4 & 27.4 & \\
V087 & - & $\mathrm{x}$ & -149.6 & 25.0 & $\mathrm{dm} f_{0.61}$ \\
V088 & $\mathrm{x}$ & - & -150.5 & 10.8 & \\
V097 & $\mathrm{x}$ & $\mathrm{x}$ & -151.1 & 24.0 & $f_{0.61}$ \\
V107 & $\mathrm{x}$ & $\mathrm{x}$ & -145.7 & 29.5 & \\
V125 & $\mathrm{x}$ & - & -145.8 & 22.9 & $\mathrm{dm} f_{0.61}$ \\
V140 & - & $\mathrm{x}$ & -160.0 & 30.0 & $\mathrm{Bl}$ \\
V203 & $\mathrm{x}$ & - & -151.1 & 10.4 & $\mathrm{Bl}$ \\
New Var. & - & $\mathrm{x}$ & -143.8 & 2.7 & \\
\hline
\end{tabular}

${ }^{a}$ Additional features of the light curve; see in Jurcsik et al. (2015).

${ }^{a}$ Data are derived from the entire Hectochelle observations.

in Jurcsik et al. 2015) are indicated in the last column of Table 5 . The $\gamma$-velocity and $A_{\mathrm{RV}}$ correspond to the $a_{0}$ term and to the peak to peak amplitude according to an appropriateorder Fourier fit to the RV time-series, respectively. The Hectochelle@MMT RV data are about 10 times more accurate than the Hydra@WIYN results, therefore, the Hydra@WIYN data are taken into account in deriving the $\gamma$ velocities and the RV amplitudes only for stars with no or incomplete phase-coverage by the Hectochelle@MMT data.

When it is possible, the mean and the amplitude of the RV curves are listed separately for the two runs of the
Hectochelle@MMT observations of Blazhko stars. Columns $4-6$ and $7-9$ in Table 7 list the $\gamma$-velocity and $A_{\mathrm{RV}}$ derived from different parts of the observations, separately. See also the footnotes of Table 7 for further details.

The rms scatter of the RV curves of single-mode RRLs in the Hectochelle@MMT data is typically 0.5 $0.7 \mathrm{~km} \mathrm{~s}^{-1}$, i.e., apart from the systematic errors, the mean and the amplitude of the RV curves are determined with about 0.1 and $0.3 \mathrm{~km} \mathrm{~s}^{-1}$ accuracy, respectively, if the phase coverage is complete. 16 of the 79 variables observed spectroscopically have only Hydra@WIYN measurements. Taking into account the $5-7 \mathrm{~km} \mathrm{~s}^{-1}$ uncertainty of these RV data, and the number of observations available for the variables $(60-80)$, the mean RV values are determined with $0.5-0.8 \mathrm{~km} \mathrm{~s}^{-1}$ accuracy for these stars.

The uncertainties of the mean and the amplitude values for variables with improper phase coverage are larger than estimated for the other stars.

There are two stars, V115 and V123, for which positioning was not accurate enough. Consequently, the Hectochelle@MMT spectra of these stars have smaller S/N ratio than for the other stars and the rms scatter of their derived RV curves is $2-3 \mathrm{~km} \mathrm{~s}^{-1}$.

The differences between the mean RVs determined at the different-amplitude phases of the modulation of at least 4 Blazhko stars (V035, V066, V104 and V130) seem to be larger than that the uncertainties would indicate. The absolute value of the mean RV is higher at the large-amplitude phase than at the small-amplitude one in each of these cases. We do not think, however, that the differences in the mean RVs of Blazhko stars at different phases of the modulation would indeed mean real changes of the $\gamma$-velocity of the stars. Instead, most probably, the dynamics of the atmo- 


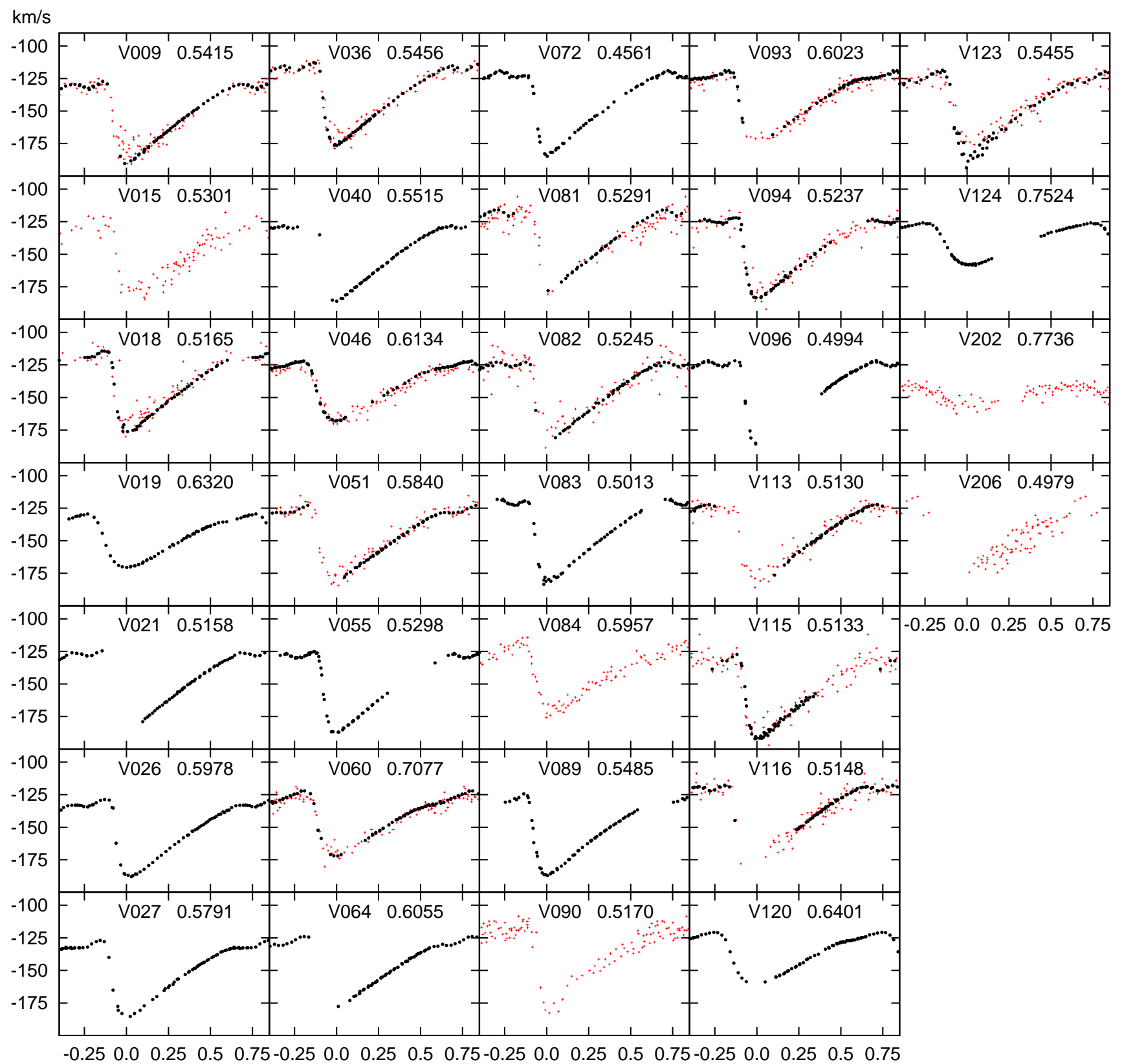

Figure 3. Radial-velocity curves of stable-light-curve RRab stars in M3. Symbols and labels are as given in the caption of Fig. 2.

sphere produce these apparent discrepancies, at least partly. The RV is determined from metallic lines, which are formed at different depth in different phases of the pulsation. However, the motion of the atmosphere is not homogeneous, and strong velocity gradients appear not only during the most violent phases of the pulsation (Chadid \& Preston 2013; Gillet \& Fokin 2014). As a consequence, the mean of the derived RVs does not necessarily reflect the real $\gamma$-velocity of the star.

Moreover, despite the strong line doubling, which characterises the spectra during the brightening phase of the pul- sation, making these RVs doubtful (read also Preston 2011, for a detailed discussion on the atmospheric dynamics of RRL stars), these RVs are also counted when calculating the mean. As these effects may be amplitude dependent, they can explain the apparent connection between the amplitude and the sign of the bias in the mean RVs of Blazhko stars.

Another source of an amplitude-dependent bias on the RVs and on their mean is in the selection of the synthetic template spectrum for the reduction process (described in Sect. 2.2.1). It was found that the amplitude of the deduced 


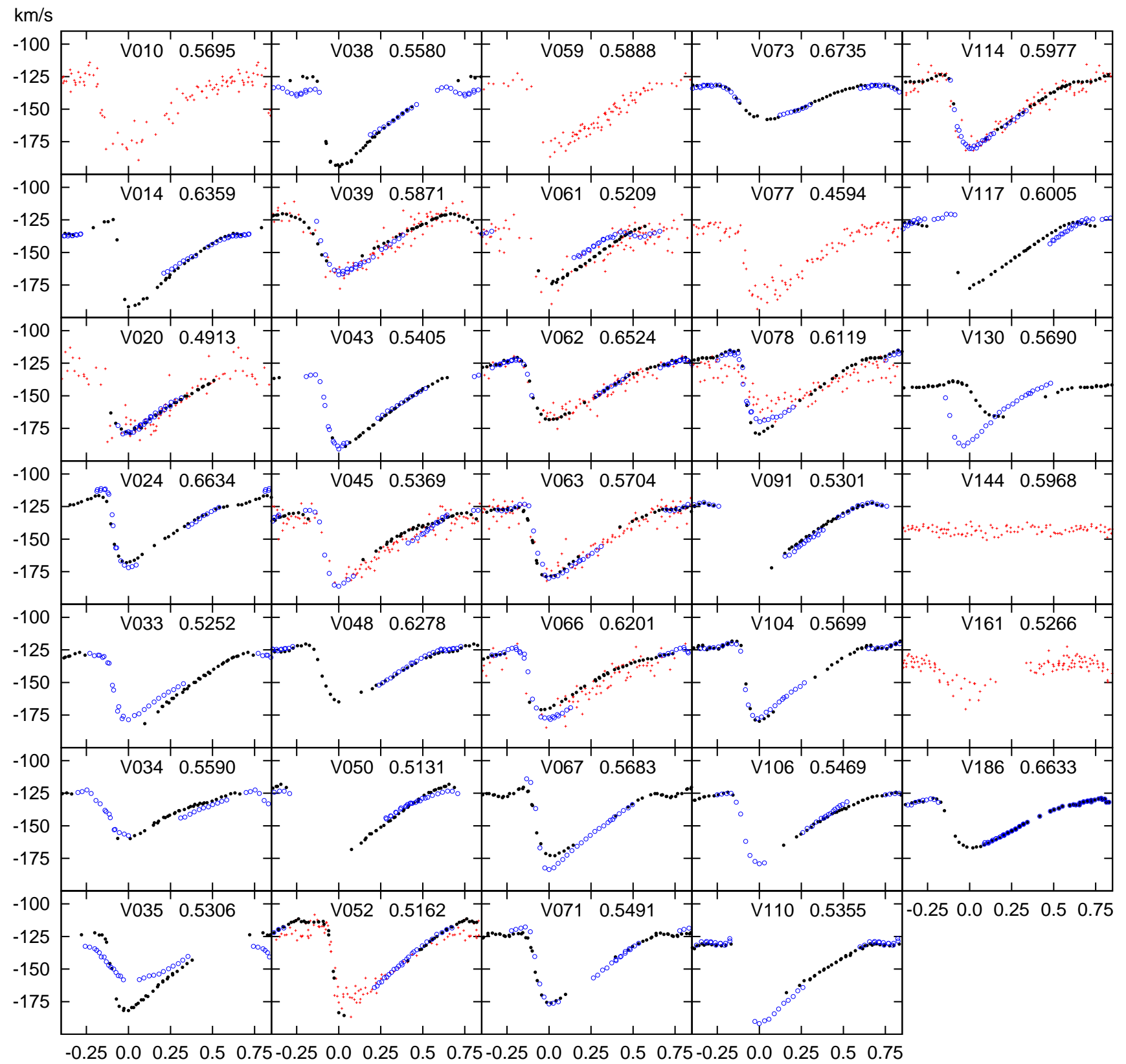

Figure 4. Radial-velocity curves of Blazhko RRab stars in M3. Symbols and labels are as given in the caption of Fig. 2.

RV curve depends, in some extent, on the temperature of the template used. The amplitude of the $T_{\text {eff }}$ variation during the pulsation of RRL stars can be as large as $2000 \mathrm{~K}$. Therefore, using a fixed-temperature template to derive the CCF of the spectra, an amplitude-dependent bias of the results is generated.

\subsection{A new overtone variable}

Spectra of non-variable stars were also observed with the Hectochelle@MMT for calibration purposes. The RV data of one of these stars turned out to show very small-amplitude, short-period RV variation. The new variable is at a large, 24.5 arcmin radial distance from the cluster centre, at the position of 13:41:18.85 +28:01:57 (J2000). The magnitudes show periodic variation with $\mathrm{a} \sim 0.25 \mathrm{~d}$ periodicity, in accordance with the $\mathrm{RV}$ variation. The phased $B V I_{\mathrm{C}}$ light curves and the RV data of the new variable are shown in Fig. 5. The mean $B, V$ and $I_{\mathrm{C}}$ magnitudes (15.90, 15.68 and $15.45 \mathrm{mag}$, respectively) of the star fit the blue edge of the instability strip of overtone variables on the horizontal branch (cf. Fig. 3 in Jurcsik et al. 2015). Its very 
Table 6. Summary of the radial-velocity data of stable-lightcurve RRab stars in M3.

\begin{tabular}{lcccc}
\hline Star $^{a}$ & \multicolumn{2}{c}{ Observation } & $\gamma$ & $A_{\mathrm{RV}}$ \\
& Hydra & Hect. & {$\left[\mathrm{km} \mathrm{s}^{-1}\right]$} & {$\left[\mathrm{km} \mathrm{s}^{-1}\right]$} \\
\hline V009 & $\mathrm{x}$ & $\mathrm{x}$ & -152.6 & 63.3 \\
V015 & $\mathrm{x}$ & - & -149.0 & 61.7 \\
V018 & $\mathrm{x}$ & $\mathrm{x}$ & -140.2 & 62.8 \\
V019 & - & $\mathrm{x}$ & -148.9 & 40.9 \\
V021 & - & $\mathrm{x}$ & $-149.2:$ & $62.0:$ \\
V026 & - & $\mathrm{x}$ & -153.8 & 60.8 \\
V027 & - & $\mathrm{x}$ & -152.2 & 59.1 \\
V036 & $\mathrm{x}$ & $\mathrm{x}$ & -139.0 & 63.6 \\
V040 & - & $\mathrm{x}$ & -150.2 & 62.4 \\
V046 & $\mathrm{x}$ & $\mathrm{x}$ & -143.0 & 45.6 \\
V051 & $\mathrm{x}$ & $\mathrm{x}$ & -147.4 & 57.0 \\
V055 & - & $\mathrm{x}$ & -150.6 & 62.7 \\
V060 & $\mathrm{x}$ & $\mathrm{x}$ & -145.3 & 51.5 \\
V064 & - & $\mathrm{x}$ & $-148.5:$ & $55.6:$ \\
V072 & - & $\mathrm{x}$ & -145.2 & 64.2 \\
V081 & $\mathrm{x}$ & $\mathrm{x}$ & -141.5 & 63.1 \\
V082 & $\mathrm{x}$ & $\mathrm{x}$ & -147.0 & 62.8 \\
V083 & - & $\mathrm{x}$ & -143.6 & 63.1 \\
V084 & $\mathrm{x}$ & - & -143.6 & 55.4 \\
V089 & - & $\mathrm{x}$ & -151.0 & 63.7 \\
V090 & $\mathrm{x}$ & - & -140.1 & 67.8 \\
V093 & $\mathrm{x}$ & $\mathrm{x}$ & -143.8 & 55.8 \\
V094 & $\mathrm{x}$ & $\mathrm{x}$ & -146.7 & 62.9 \\
V096 & - & $\mathrm{x}$ & -146.7 & 65.5 \\
V113 & $\mathrm{x}$ & $\mathrm{x}$ & -145.4 & 63.0 \\
V115* & $\mathrm{x}$ & $\mathrm{x}$ & -155.4 & 65.7 \\
V116 & $\mathrm{x}$ & $\mathrm{x}$ & $-143.6:$ & $64.5:$ \\
V120 & - & $\mathrm{x}$ & -139.7 & 39.8 \\
V123* & $\mathrm{x}$ & $\mathrm{x}$ & -147.9 & 66.2 \\
V124 & - & $\mathrm{x}$ & -141.2 & 31.8 \\
V202 & $\mathrm{x}$ & - & -148.5 & 15.2 \\
V206 & $\mathrm{x}$ & - & $-148.6:$ & $59.0:$ \\
\hline
\end{tabular}

$a *$ denotes stars with erroneous positioning.

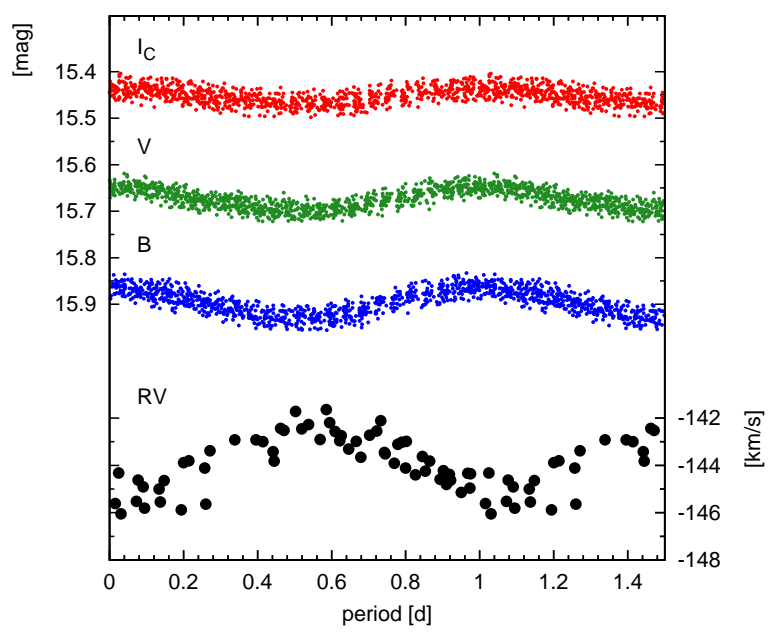

Figure 5. Phased $B V I_{\mathrm{C}}$ light curves and RV curve of the new variable.
Table 7. Summary of the radial-velocity data of Blazhko RRab stars in M3.

\begin{tabular}{|c|c|c|c|c|c|c|c|c|}
\hline \multirow[t]{2}{*}{ Star } & \multirow{2}{*}{\multicolumn{2}{|c|}{$\begin{array}{c}\text { Instr. } \\
\text { Hyd.Hect. }\end{array}$}} & \multicolumn{3}{|c|}{$\begin{array}{c}\gamma^{*} \\
{\left[\mathrm{~km} \mathrm{~s}^{-1}\right]}\end{array}$} & \multicolumn{3}{|c|}{$\begin{array}{c}A_{\mathrm{RV}}^{*} \\
{\left[\mathrm{~km} \mathrm{~s}^{-1}\right]}\end{array}$} \\
\hline & & & all & Hect1 & Hect2 & all & Hect1 & Hect2 \\
\hline V010 & $\mathrm{x}$ & - & -148.3 & & & 60.6 & & \\
\hline V014 & - & $\mathrm{x}$ & & -153.7 & & & 63.6 & \\
\hline V020 & $\mathrm{x}$ & $\mathrm{x}$ & -151.0 : & & & 48.0: & & \\
\hline V024 & - & $\mathrm{x}$ & & -139.0 & -138.6 & & 53.4 & 60.0 \\
\hline V033 & - & $\mathrm{x}$ & & & -148.2 & & & 51.7 \\
\hline V034 & - & $\mathrm{x}$ & & -141.3 & -141.8 & & 36.0 & 37.4 \\
\hline V035 & - & $\mathrm{x}$ & & -145.5 & -144.0 & & 59.6 & 27.8 \\
\hline V038 & - & $\mathrm{x}$ & & -154.9 & & & 69.2 & \\
\hline $\mathrm{V}^{2} 39^{a}$ & $\mathrm{x}$ & $\mathrm{x}$ & -140.0 & & & 40.9: & & \\
\hline V043 & - & $\mathrm{x}$ & -156.3 & & & 56.0 & & \\
\hline V045 & $\mathrm{x}$ & $\mathrm{x}$ & & & -153.4 & & & 58.9 \\
\hline V048 & - & $\mathrm{x}$ & & -141.4 & & & 45.2 & \\
\hline V050 & - & $\mathrm{x}$ & $-145:$ & & & $>50$ & & \\
\hline V052 & $\mathrm{x}$ & $\mathrm{x}$ & & -141.6 & & & 75.0 & \\
\hline V059 & $\mathrm{x}$ & - & -150.5 & & & 55.0 & & \\
\hline V061 & $\mathrm{x}$ & $\mathrm{x}$ & & $-148:$ & & & 46.0: & \\
\hline $\mathrm{V} 062^{a}$ & $\mathrm{x}$ & $\mathrm{x}$ & -143.5 & & & 47.0 & & \\
\hline V063 & $\mathrm{x}$ & $\mathrm{x}$ & & -147.8 & -147.7 & & 52.7 & 58.6 \\
\hline V066 & $\mathrm{x}$ & $\mathrm{x}$ & & -147.0 & -149.4 & & 43.7 & 54.1 \\
\hline V067 & - & $\mathrm{x}$ & & -144.0 & $-143.3:$ & & 52.5 & 70.5: \\
\hline V071 & - & $\mathrm{x}$ & & -143.9 & -143.6 & & 53.2 & 60.0 \\
\hline V073 & - & $\mathrm{x}$ & & -143.2 & -143.4 & & 26.4 & 24.0 \\
\hline V077 & $x$ & - & -152.4 & & & 57.0 & & \\
\hline V078 & $\mathrm{x}$ & $\mathrm{x}$ & & -142.4 & -142.6 & & 65.4 & 53.4 \\
\hline V091 & - & $\mathrm{x}$ & $-145:$ & & & $>55$ & & \\
\hline V104 & - & $\mathrm{x}$ & & -143.7 & -143.1 & & 63.4 & 61.1 \\
\hline V106 & - & $\mathrm{x}$ & & & -146.9 & & & 53.5 \\
\hline V110 & - & $\mathrm{x}$ & & & -153.1 & & & 67.1 \\
\hline $\mathrm{V} 114^{a}$ & $\mathrm{x}$ & $\mathrm{x}$ & -147.8 & & & 58.4 & & \\
\hline V117 & - & $\mathrm{x}$ & & -147.0 : & & & $50.2:$ & \\
\hline $\mathrm{V} 130^{b}$ & - & $\mathrm{x}$ & & -150.8 & $-154.5:$ & & 27.3 & 52.3 \\
\hline & & & & - & $-152.8:$ & & & 58.2 \\
\hline $\mathrm{V} 144^{c}$ & $\mathrm{x}$ & - & -143.1 & & & 2 & & \\
\hline V161 & $\mathrm{x}$ & - & -142.7 & & & 23 & & \\
\hline V186 & - & $\mathrm{x}$ & -146.9 & & & 38.0 & & \\
\hline
\end{tabular}

* all: according all the RV data;

* Hect1: the first run of the Hectochelle observations.

* Hect2: the second run of the Hectochelle observations.

${ }^{a}$ Data are derived from the entire Hectochelle observations.

${ }^{b}$ Two solutions are given for the scarce large-amplitude phase.

${ }^{c}$ Large-amplitude modulation on a very long timescale.

small amplitude, short period, and blue colour indicate that the new variable is a second-overtone RRL belonging to M3. Its $\gamma$-velocity $\left(-143.8 \mathrm{~km} \mathrm{~s}^{-1}\right)$ and proper motion $\left(\mu_{\alpha} \cos \delta=-1.9\right.$ mas $\mathrm{yr}^{-1}, \mu_{\delta}=-0.5$ mas $\mathrm{yr}^{-1}$ Smart \& Nicastro 2014) agree with the mean cluster velocity $\left(-147 \mathrm{~km} \mathrm{~s}^{-1}\right.$, see in Sect. 5$)$ and absolute proper motion $\left(\mu_{\alpha} \cos \delta, \mu_{\delta}=-2.5 \pm 0.6,-2.1 \pm 0.8\right.$ mas yr $^{-1}$, Tucholke et al. 1994) reasonably well. 

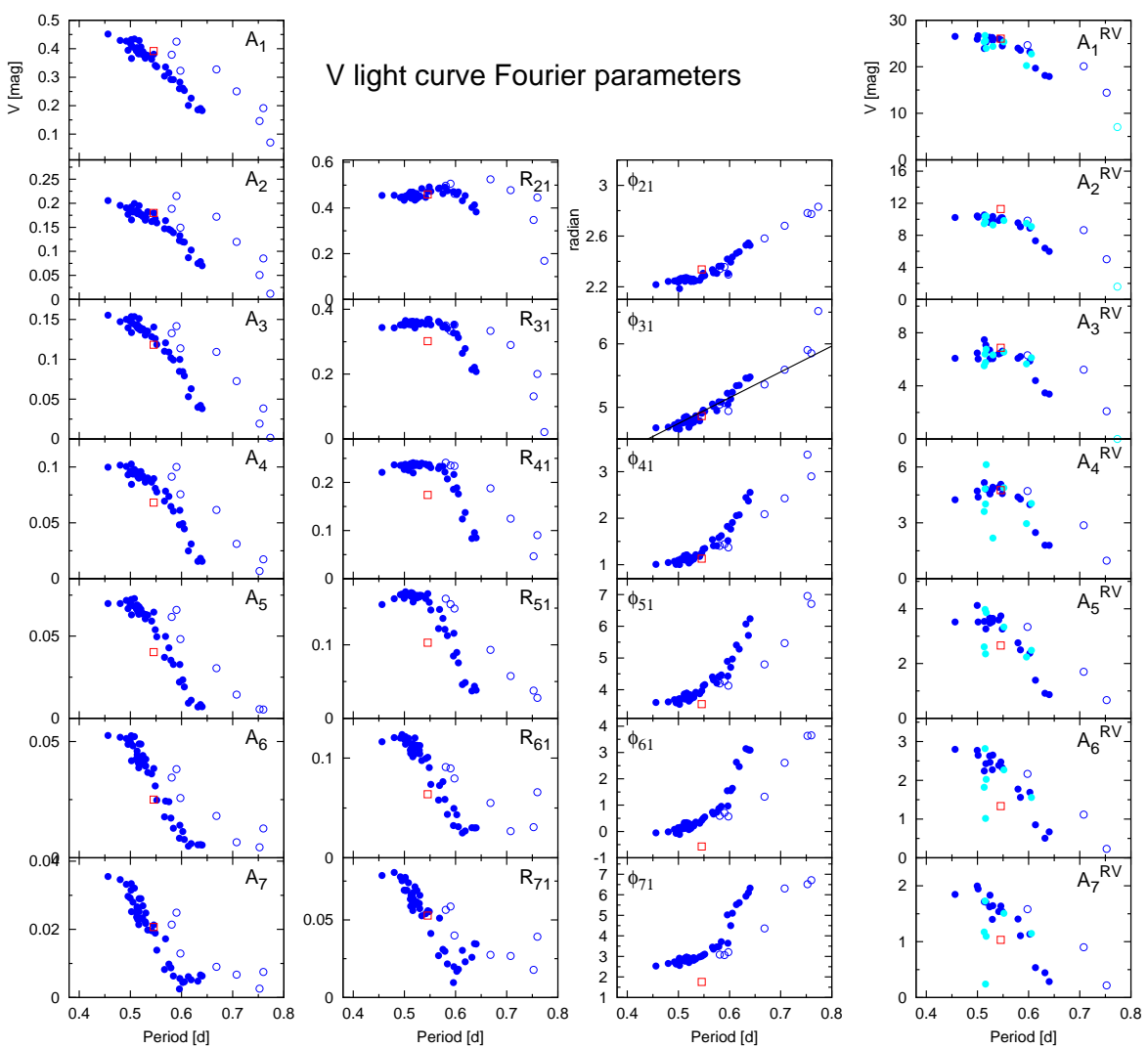

\section{$\mathrm{RV}$ curve Fourier parameters}
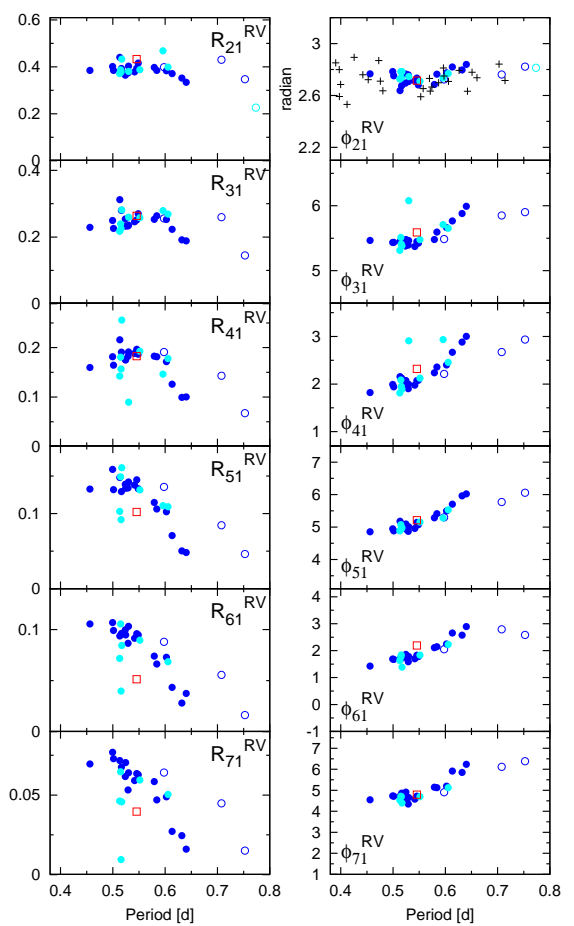

Figure 6. The Fourier parameters of the $\mathrm{V}$ light curves and the radial-velocity curves of stable RRab stars in M3 are shown in the left- and right-hand panels, respectively. The Fourier amplitudes, amplitude ratios, and epoch-independent phase differences of sin decomposition $\left(A_{1}-A_{7}, R_{21}-R_{71}\right.$ and $\left.\Phi_{21}-\Phi_{71}\right)$ are plotted. Open symbols denote OoII variables. The light-blue symbols in the plots of the RV data denote uncertain values, variables with noisy and/or scarce RV data. The $\Phi_{21}{ }^{R V}$ values of Galactic field RRab stars are also shown for comparison (black crosses in the top/right-hand panel). Note that the scales of the $R_{k 1}$ and $R_{k 1}{ }^{\mathrm{R} V}$ and the $\Phi_{k 1}$ and $\Phi_{k 1} \mathrm{RV}$ amplitude-ratio and phase-difference panels are identical, respectively, for a better comparison. The line drawn in the Period $-\Phi_{31}$ panel corresponds to $[\mathrm{Fe} / \mathrm{H}]=-1.45$ according to the metallicity formula derived in Jurcsik \& Kovács (1996). Results for the peculiar V123 are shown by red square symbols.

Table 8. Comparison of the statistics of the $\Phi_{21} \mathrm{RV}$ phase differences of the radial-velocity curves of Galactic field and M3 RRab stars.

\begin{tabular}{cccccc}
\hline & & \multicolumn{3}{c}{$\Phi_{21} \mathrm{R} V$} \\
Sample & $\mathrm{N}$ & min. & $\max$. & mean & s.d. \\
\hline M3 & 31 & 2.64 & 2.84 & 2.74 & 0.05 \\
field & 26 & 2.53 & 2.89 & 2.72 & 0.09 \\
\hline
\end{tabular}

\section{FOURIER PARAMETERS OF THE $V$ LIGHT CURVE AND THE RADIAL-VELOCITY CURVE OF STABLE RRAB STARS}

The Fourier parameters of the light curves of RRL stars are good indicators of the physical properties of the stars (Simon \& Teays 1982; Jurcsik \& Kovács 1996; Jurcsik 1998; Kovács \& Walker 2001), but systematic studies of the Fourier parameters of the RV curves are available only for Cepheids (Moskalik et al. 2000; Pont et al. 2001). Liu (1991) has found that, in contrast with the large diversity of the shapes of the light curves, the shapes of the available sample of RRL RV curves is relatively stable; actually only their amplitudes are scaled according to the amplitude of the light curve.

The Fourier parameters of the $V$ light-curves and the RV curves of stable RRab stars in M3 are compared in Fig. 6. The light-curve parameters follow the known, period dependent trends, and the positions of the OoI and OoII stars are well separated in the panels showing the amplitudes, amplitude ratios and the higher-order phase differences. In contrast, the OoI and OoII variables follow the same tracks in the panels showing the lower-order phase differences. Among the Fourier parameters of the light curve shown, the $\Phi_{31}$ data define the most linear relation with the period, in accordance with the metallicity formula (Jurcsik \& Kovács 1996, JK96), which predicts a linear relation between these parameters for a sample of different period variables of the same metallicity. The straight line in this panel corresponds to the JK96 relation for $[\mathrm{Fe} / \mathrm{H}]=-1.45$. The only star, which deviates from this relation significantly, is the longest period, smallest amplitude, OoII star, V202. The light curve of this star is sinusoidal, consequently its 3rd-order Fourier parameters are uncertain.

The amplitudes and amplitude ratios of the RV curves follow the same trends as the Fourier parameters of the light curve. Outliers are only among the poor-quality RV data 
Table 9. Radial variation of the dispersion of the mean radial velocities of RR Lyrae stars and giants in M3.

\begin{tabular}{lrrrcc}
\hline $\begin{array}{l}\mathrm{RD}^{a} \\
\text { arcmin }\end{array}$ & $\mathrm{N}$ & $\begin{array}{r}\text { mean RD } \\
\text { arcmin }\end{array}$ & s.d. & $\begin{array}{c}\text { mean RV }(\gamma \text {-vel. }) \\
\mathrm{km} / \mathrm{s}\end{array}$ & s.d. \\
\hline $\begin{array}{l}\text { RR Lyrae } \\
0.0-2.5\end{array}$ & 14 & 1.72 & 0.43 & -149.14 & 5.85 \\
$2.5-5.3$ & 32 & 3.71 & 0.87 & -145.95 & 4.75 \\
$5.3-7.5$ & 13 & 6.41 & 0.66 & -146.99 & 3.65 \\
$7.5-12.3$ & 16 & 9.24 & 1.43 & -145.72 & 2.37 \\
$12.3-$ & 4 & 21.15 & 6.78 & -148.90 & 4.81 \\
giants & & & & & \\
$0.0-2.5$ & 15 & 1.63 & 0.61 & -147.81 & 7.62 \\
$2.5-5.3$ & 26 & 3.71 & 0.76 & -147.22 & 4.88 \\
$5.3-7.5$ & 12 & 6.59 & 0.46 & -149.65 & 4.05 \\
$7.5-12.3$ & 48 & 9.93 & 1.22 & -147.39 & 2.61 \\
$12.3-$ & 53 & 18.08 & 4.64 & -146.41 & 2.75 \\
\hline
\end{tabular}

a Projected radial distance.

shown by light-blue symbols in Fig. 6. What is, however, indeed surprising, is the behaviour of the phase differences of the RV curves. The full range of the variation of the $\Phi_{21}$ $\ldots \Phi_{71}$ phase differences increases from 0.65 to 4.45 for the light-curve parameters, but only from 0.20 to 2.03 for the $\Phi_{k 1} \mathrm{RV}$ parameters. (Note that, for a better comparison, the scales of the amplitude-ratio and phase-difference plots for the $V$ and RV data are identical.)

Considering $\Phi_{21}{ }^{R V}$, no period dependence of this parameter is detected at all. To check the stability of the $\Phi_{21}{ }^{R V}$ phase differences of the RV curves of RRab star, the data of Galactic field stars compiled by Kovács (2003) are also shown in this panel by crosses. The $[\mathrm{Fe} / \mathrm{H}] \mathrm{s}$ of these field stars cover a wide metallicity range: from solar (0.0) to very metal poor $(-1.9)$. The $[\mathrm{Fe} / \mathrm{H}]$ of each field star with a period shorter than $0.5 \mathrm{~d}$ is larger than -1.0. In spite of the significant differences of the field and M3 samples, their $\Phi_{21}{ }^{\mathrm{R} V}$ phase differences seem to have very similar values. The statistics of the $\Phi_{21}{ }^{R V}$ phase differences of the two samples are summarised in Table 8 .

We thus conclude that the $\Phi_{21} \mathrm{R} V$ phase difference of the RV curve of RRab stars is uniformly constant, it does not show any detectable period or metallicity dependence larger than the uncertainty of the data.

According to $Z=0.001, M=0.65 M_{\odot}$ and $L=52.5 L_{\odot}$ convective, nonlinear pulsation models with periods between $0.48-0.80 \mathrm{~d}$ (Feuchtinger 1999) the $\Phi_{21}{ }^{\mathrm{R} V} \ldots \Phi_{51} \mathrm{R} V$ phase differences of model RV curves vary within about twice as large ranges as observed in M3, from $0.5\left(\Phi_{21}{ }^{R V}\right)$ to 2.0 $\left(\Phi_{51}{ }^{R V}\right)$, and constancy of the $\Phi_{51} R V$ phase difference is predicted in the $0.45-0.70 \mathrm{~d}$ period interval, which does not seem to be valid based on the observations.

\section{RADIAL DEPENDENCE OF THE DISPERSION OF THE MEAN RADIAL VELOCITIES OF RR LYRAE STARS}

The statistics of the radial dependence of the dispersion of the RV data in GCs is a key function for dynamical studies. A large sample of RV data of M3 giants, observed also with the Hechtochelle@MMT instrument, were published recently

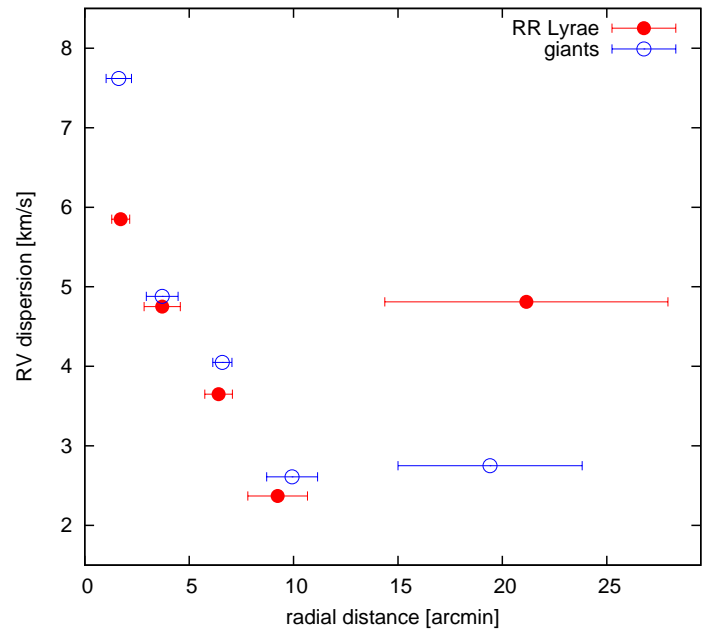

Figure 7. Comparison of the projected radial dependence of the dispersion of the $\gamma$-velocities of RR Lyrae stars and the radial velocities of giants (Kimmig et al. 2015) in M3. The points are shown at the mean value of the radial distances of the sub-samples and the horizontal bars denote their standard deviations.

by Kimmig et al. (2015). Although other RV studies of M3 are also available in the literature (e.g., Gunn \& Griffin 1979; Soderberg et al. 1999; Pilachowski et al. 2000; Smolinski et al. 2011; Kamann et al. 2014), these studies include smaller number of objects, and there is some overlap between the sample stars in some cases. Therefore, we selected exclusively the Kimmig et al. (2015) data for the comparison of the RV dispersion values of RRL and of other giant stars in M3. The typical accuracy of the RV values given by Kimmig et al. (2015) is some tenths of $\mathrm{km} \mathrm{s}^{-1}$.

The mean value of the $\gamma$-velocities of the $79 \mathrm{RRL}$ stars listed in Table 5, 6 and 7 is $-146.8 \mathrm{~km} \mathrm{~s}^{-1}$ with $4.52 \mathrm{~km} \mathrm{~s}^{-1} \mathrm{rms}$. If multiple entries of the mean RV of Blazhko stars are given in Table 7, the average of these values are taken here. This result is in good agreement with the $-147.4 \mathrm{~km} \mathrm{~s}^{-1}$ mean RV with $4.05 \mathrm{~km} \mathrm{~s}^{-1} \mathrm{rms}$ of the 139 giants (Kimmig et al. 2015). The minor, $0.5 \mathrm{~km} \mathrm{~s}^{-1}$ smaller RV dispersion value of the Kimmig's data may be explained by the overpopulation of the sample stars in the outer regions in this data-set. While there are similar number of stars in the inner regions (projected radial distances $\mathrm{RD}<12$ arcmin) in the samples of RRL stars and Kimmig's giants, the two samples contain quite different number of stars in the outer regions. Only a few RRL but about half of the total sample of the giants are located here and the RV dispersion is known to be decreasing outwards in GCs.

Other RV measurments of M3 stars are also in good agreement with the above results, e.g. Gunn \& Griffin (1979) determined $-146.9 \mathrm{~km} \mathrm{~s}^{-1}$ cluster-mean velocity for a sample of 111 stars, while Soderberg et al. (1999) derived $-147.0 \mathrm{~km} \mathrm{~s}^{-1}$ for 87 giants.

The radial dependence of the statistics of the mean RVs of the RRL stars (Table 5, 6, 7) and of the Kimmig et al. (2015) sample are summarised in Table 9 and the results are shown in Fig. 7. The range of the projected RDs, the number of stars within the RD range $(\mathrm{N})$, the mean and the 
rms of the RDs and the mean and the rms of the mean RVs within the given RD bin are listed in the $1-6$ columns of Table 9, respectively. The accepted binning was chosen to obtain the possible most similar $\mathrm{N}$ and mean $\mathrm{RD}$ values for the four inner bins of the two samples.

The RV dispersion of RRL stars is smaller than the RV dispersion of giants in the four inner bins as can be seen in Fig. 7. However, the outermost bin contains only 4 RRL stars but 53 giants; thus the dispersions of the mean RVs of the two samples cannot be compared reliably here. As the mean RV can be determined less accurately for pulsating variables than for constant stars (see details in Sect. 2.2 and 3.2) this is just the opposite of what one would expect. Therefore, the statistical significance of the differences has to be checked to decide the reliability of this result.

To test the significance of the apparent systematic differences between the two samples we use a simple, two-parameter fit to the data as defined in eq. 12. of Hernandez \& Jimènez (2012) instead of a physically motivated more model-specific solution (e.g., King 1966). The individual errors of the RV values are not taken into account in the fitting process, because they cannot be determined correctly for RRL stars if their phase coverage is not complete and because of the detected amplitude-dependent bias of the mean RVs. However, this is not relevant for our purpose, since the error of the mean RVs are supposed to be still significantly smaller than the intrinsic RV dispersion, especially in the inner RD bins.

We made the null hypothesis that the effect is purely accidental. Supposing this null hypothesis is valid, we merged the RRL and giant star data into one sample and fitted the radial dependence of the velocity standard deviation using eq. 12. of Hernandez \& Jimènez (2012)

$\sigma(r)=\sigma_{1} \exp \left(-\frac{r^{2}}{r_{\sigma}^{2}}\right)+\sigma_{0}$

where $\sigma_{1} . r_{\sigma}$, and $\sigma_{0}$ are constants determined by least squares fitting. Applying this formula, we computed the standardized sum of squares of the velocity differences by

$Q^{2}=n^{-1} \sum_{i=1}^{n} \frac{\left(R V_{i}-R V_{\text {mean }}\right)^{2}}{\sigma\left(r_{i}\right)^{2}}$

for the RRL and the giant stars separately ( $n$ is the sample size in the above formula). If the null hypothesis is valid, the $Q_{\mathrm{RRL}}{ }^{2} / Q_{\text {giants }}{ }^{2}$ ratio of the RRL and giant stars should not differ significantly from unity. In our case the value of this ratio is 0.82 . Assuming a valid null hypothesis the probability for getting this value purely accidentally is $p=0.18$. Consequently, the apparent difference between the standard deviations of the RRL and giant stars is not, in fact, significant statistically.

\section{ANALYSIS OF THE COMBINED PHOTOMETRIC AND RADIAL-VELOCITY DATA OF STABLE-LIGHT CURVE RR LYRAE STARS IN M3}

\subsection{Parallel light and radial-velocity curves}

The simultaneous photometric light/colour curves and RV data used in the analysis are calculated from appropriate order $(3-16)$ harmonic fits to the observations. The photometric data of overtone and double-mode variables were published in Jurcsik et al. (2015).

When the Hydra@WIYN RVs are used in combination with the photometry, the phase-match of the data are guaranteed by harmonizing the phases of the 2012 light curves with the photometric observations obtained in $1998-1999$ (Benkő et al. 2006; Jurcsik et al. 2012).

\subsection{Baade-Wesselink analysis}

The RV and photometric data of M3 discussed in Sect. 2 and published in Jurcsik et al. (2015) provide the first possibility to perform classical BW analysis of a large sample of stars in a GC.

As the tidal radii of GCs are not significantly larger than 100 pc [Navin et al. (2016) and Harris (1996) gave 113 and 85 pc values for M3, respectively], the distances of the variables should have to be the same within about a $100-200$ pc range in a given GC. Hence, the real star by star differences between the distances are expected to be smaller than the inherent $\sim 5-10$ percent uncertainty of the photometric BW method. Therefore, the analysis is performed in two steps: first, the distances of the variables with the best-accuracy and phase-coverage RV curves are determined, then, secondly, fixing the distance to the mean value of the distances of these stars, the mean radius and the radius variation are determined for the full sample of the variables observed both photometrically and spectroscopically. It was shown in Jurcsik et al. (2015) that a small-amplitude signal appears in the spectra of many RRc stars at 0.61 frequency ratio. These frequencies have, however, mmag amplitudes only, therefore these variables are also included in the analysis.

Because of their high accuracy and simultaneity with the photometry, the Hectochelle RV curves of the variables are used, when available. The additional stars are 4 RRab (V015, V084, V090 and V202) and 2 RRc stars (V037 and V088) which have only Hydra data. Some of the Hectochelle RV curves lack a complete phase coverage; to obtain a continuous fit for these data they are complemented with artificial points according to the Hydra data and/or taking into account the shape of the light curve. The uncertainty of this process has, however, a negligible effect on the distance determination of the cluster, and on the obtained results.

\subsubsection{The method}

Supposing that the pulsation is fully radial, the equality of the photometric and spectroscopic radii is the base idea of the BW method. These radii are derived according to the following equations:

$R_{\mathrm{ph}}=d \times \theta, \quad \theta=10^{0.2\left(-\left(V_{0}+B C\right)-10 * \log \left(T_{\text {eff }}\right)+37.35\right)}$

$R_{\mathrm{sp}}=R_{0}+p \times \int\left(V_{\mathrm{rad}}-\gamma\right)$,

where $R_{\mathrm{ph}}$ and $R_{\mathrm{sp}}, d, \theta, V_{0}$, B.C., $R_{0}, p$ and $\gamma$ are the photometric and spectroscopic radii, the distance, the stellar angular radius, the $V$ magnitude corrected for interstellar absorption, the bolometric correction, the mean radius, the projection factor and the mean value of the RV, respectively. Note that, instead of $\mathrm{AU}$ and $\mathrm{pc}$, the dimensions of 
$[\mathrm{t}]$
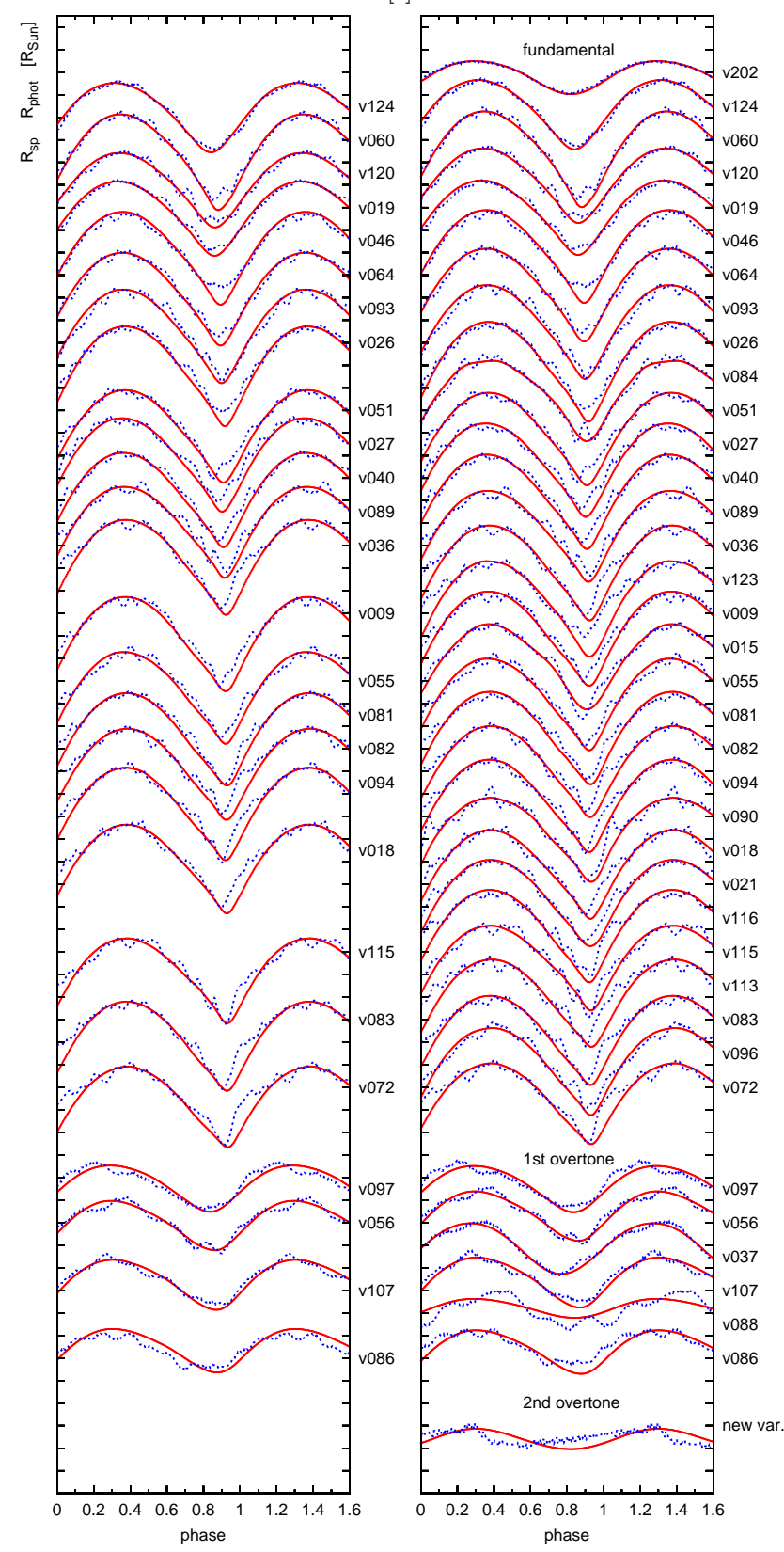

Figure 8. Comparison of the photometric $\left(R_{\mathrm{phot}}\right.$ : blue dotted lines) and spectroscopic $\left(R_{\mathrm{sp}}\right.$ : red continuous lines) radii variation of single-mode RR Lyrae stars in M3. The best-fit solutions with the distance as a free parameter are shown in the left-hand panel for variables with complete, high accuracy photometric and radial-velocity data. Using a fixed, $10480 \mathrm{pc}$ value for the distance of M3, the matches of the $R_{\mathrm{phot}}$ and $R_{\mathrm{sp}}$ curves are shown in the right-hand panel. Stars are shown in the order of decreasing pulsation period from top to bottom. The $R$ curves are vertically shifted for clarity, the tics on the $y$ axes denote $0.20,0.10$ and $0.02 R_{\odot}$ for the fundamental, first- and second-overtone RR Lyrae stars, respectively.
$R$ and $d$ are $R_{\odot}$ and parsec in the first part of Eq. 3, thus $\theta$ is measured in $0.00456 \operatorname{arcsec}$ unit. The B.C. scale is set to give -0.07 B.C. $\odot$, and $M_{\mathrm{bol} \odot}=4.74 \mathrm{mag}$ is regarded (Torres 2010). The value of the $p$-factor is taken to be 1.35 (Nardetto et al. 2004). The effective temperature $\left(T_{\text {eff }}\right)$ and B.C. are determined from the dereddened $\left(V-I_{\mathrm{C}}\right)$ colour and the effective $\log g$, using synthetic $\left(V-I_{\mathrm{C}}\right)$ colours of static atmosphere models (Castelli \& Kurucz 2003). The effective $\log g$ is calculated as the sum of the static $\log g$ according to eq. 15. in Jurcsik (1998), which yields $\log g=2.7-2.9$ values for RRab stars, and the derivative of the RV curve. The static $\log g$ of the overtone variables is taken to be 3.0. The metallicity and reddening of M3 are adopted to be -1.5 and $E(B-V)=0.014 \mathrm{mag}$, respectively (Harris 1996). The enhancement of the $\alpha$ elements is supposed to be 0.3 dex (Carney 1996). The static atmosphere model grids are interpolated to obtain 0.05 and $5 \mathrm{~K}$ resolution in $\log g$ and in $T_{\text {eff }}$, respectively.

The distance and the mean radius are determined by matching the amplitudes and the zero points of the $R_{\mathrm{ph}}$ and the $R_{\mathrm{sp}}$ curves between pulsation phases $0.1 / 0.2$ and 0.8/0.9 for fundamental mode and for RRc stars showing a pronounced bump on the light curve preceding maximum brightness and by using the complete $R$ curves for overtone variables with sinusoidal light curves. For a detailed discussion on the effect of different pulsation phases on the results of BW analyses read e.g. Cacciari et al. (1992) and Jones et al. (1992).

\subsubsection{The first step: determination of the distance}

There are 22 RRab and 4 RRc stars with high S/N RV data of good phase coverage, which are appropriate for the complete analysis. The distances derived for these stars are listed in the third column of Table 10; the first and second columns list the ID of the star and the pulsation period, respectively. The $R_{\mathrm{ph}}$ and $R_{\mathrm{sp}}$ curves of the best fit for these 26 stars are shown in the left-hand panel of Fig. 8. The match between $R_{\mathrm{ph}}$ and $R_{\mathrm{sp}}$ is satisfactory; significant differences between the two curves appear only between phases 0.9 and 1.1, when the dynamics of the atmosphere is the most violent.

BW distance moduli were determined previously for only a very limited number of stars in the M4, M5, M92 and 47 Tuc GCs with 0.15 - 0.20 mag uncertainty (Cohen 1992; Liu \& Janes 1990b; Storm et al. 1994a,c). The corresponding uncertainties of the distances are $5-10$ percent. The BW distances of the $26 \mathrm{M} 3$ stars cover the $9680-11420$ pc range with $10480 \mathrm{pc}$ value of both the mean and the median. The standard deviation of the derived distances is $310 \mathrm{pc}$, i.e. about 3 percent of the mean value.

The scatter of the distance estimates reflects primarily the photometric uncertainties and the uncertainty of the fitting process of the $R_{\mathrm{ph}}$ and $R_{\mathrm{sp}}$ curves. The magnitude zero point (and amplitude) even of very-small-scatter light curves may be uncertain by $0.01-0.05 \mathrm{mag}$ in crowdedfield photometry. A $0.02 \mathrm{mag}$ error of the magnitude zero point of a given star results in about 300 pc difference of the derived distance. Even larger, about 500 pc difference in the distances may arise depending on that which part of the radii curves around the rising branch of the light curve is omitted from the fit. This is because of the inadequacy of both the photometric and spectroscopic data to derive the 


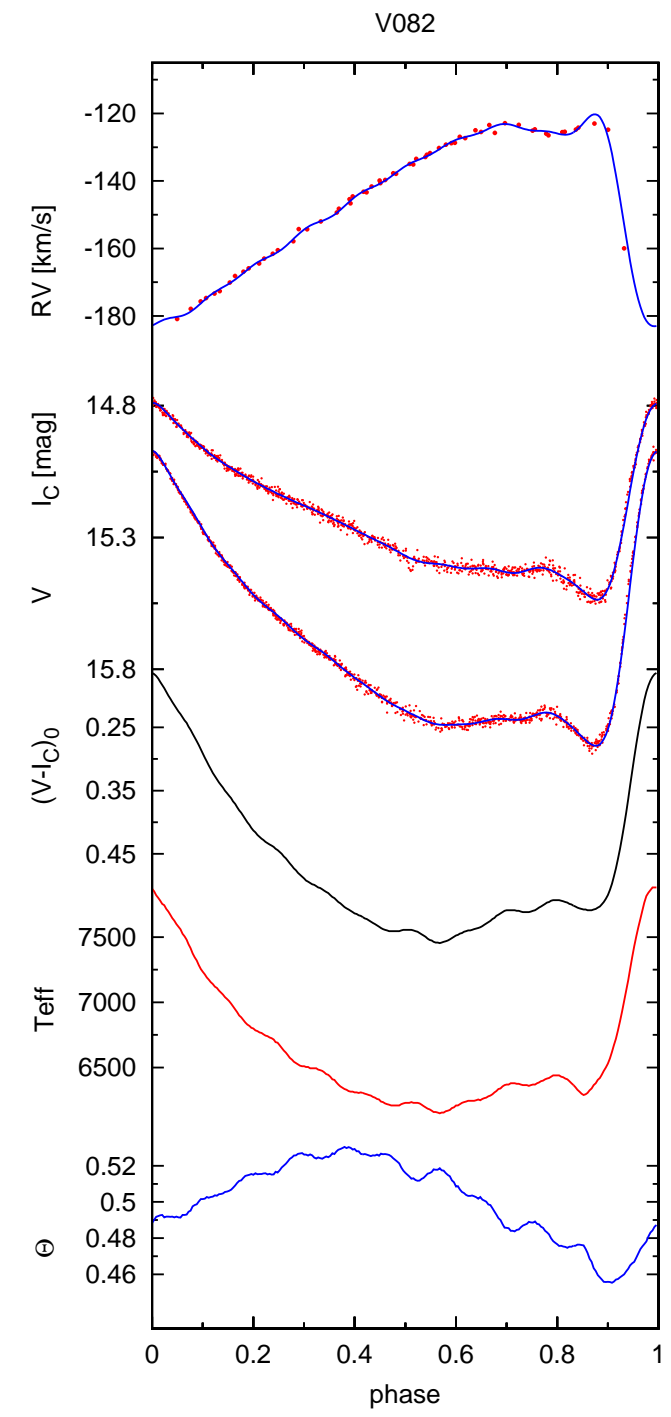

Figure 9. The measured and fitted radial velocity, $V$ and $I_{\mathrm{C}}$ light curves, the $(V-I)_{0}$ colour curve calculated according to the Fourier fits of the light curves, the corresponding temperature, and angular radius variation (in 0.00465 mas units) of V082 are shown in the figure.

radius variation during the most violent phases of the pulsation and also because of the wiggles on the photometric radius curves. These wiggles originate form the uncertainty of the process: the $V-I_{\mathrm{C}}$ colour curve is determined as the subtraction of the synthetic light curves derived as high order Fourier fits to the observations, and the marginal wiggles on the resultant $V-I_{\mathrm{C}}$ curve are magnified when transforming the logarithmic scale to linear. For an example, the RV, $V, I_{\mathrm{C}}, V-I_{\mathrm{C}}, T_{\mathrm{eff}}$ and angular radius variations of $\mathrm{V} 082$ are illustrated in Fig. 9.

The other sources of the errors of the distance estimates (uncertainties of the $p$-factor, the synthetic colours, B.C.,

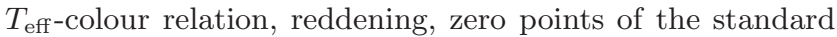
calibration of the photometry, etc.) are mostly systematic, and do not typically exceed $1-2$ percent (Marengo et al. 2004).

Consequently, the random, uncorrelated errors account for about 5 percent, while the systematic errors for $1-$ 2 percent uncertainties of the distance estimates in M3. In principle, if the errors of the 26 individual distances were perfectly uncorrelated, the accuracy of the mean distance were as good as $61 \mathrm{pc}$. Supposing that the systematic errors do not exceed 2 percent, the accuracy of distance determination of M3 is thus estimated to be $210 \mathrm{pc}$.

The average/median value of the derived distances of the 26 best, stable-light-cure RRL stars in M3 is 10480 pc, i.e. the distance modulus of the cluster is $\nu=15.10 \mathrm{mag}$. For comparison, based on the pulsation properties of RRL stars, Cassisi et al. (2001) derived 10470 pc, and the Harris (1996) catalogue gives $10200 \mathrm{pc}$ for the distance of M3. Using $K$ band period-luminosity relations Sollima et al. (2006) determined $\nu=15.07 \mathrm{mag}$, modelling the pulsation light curves of RRL stars Marconi et al. (2015) yielded 15.10 $\pm 0.1 \mathrm{mag}$ and fitting the colour-magnitude diagrams of the cluster adopting $[\mathrm{Fe} / \mathrm{H}]=-1.55$ and $[\alpha / \mathrm{H}]=0.4$ zaro-age horizontalbranch models, isochrones and evolutionary tracks $\nu=$ 15.05 - $15.04 \mathrm{mag}$ distance modulus of M3 was obtained (VandenBerg et al. 2015, 2016).

\subsubsection{The second step: the radii}

Fixing the distance of M3 to 10480 pc, the radius and the temperature variations of all the single-mode variables, which have reasonable RV curves are determined according to the process described above, however, only $R_{0}$ is determined in the fit this time.

The fits of the $R_{\mathrm{ph}}$ and $R_{\mathrm{sp}}$ curves are shown in the right-hand panel of Fig. 8 for 31 RRab, 6 first and 1 secondovertone variables assuming $10480 \mathrm{pc}$ distance for each star. The differences between the matches of the $R_{\mathrm{ph}}$ and $R_{\mathrm{sp}}$ curves according to the best fit and the fixed-distance solutions (left- and right-hand panels) are not perceivable. With the exceptions of the shortest-period overtone variables, V088, V086 and the second-overtone new variable, the match between the $R_{\mathrm{ph}}$ and $R_{\mathrm{sp}}$ curves is satisfactory for all the stars, even for those, which have only Hydra spectra or which RV curves are incomplete.

Phase differences between the $R_{\mathrm{ph}}$ and $R_{\mathrm{sp}}$ curves appear only for V086 and the new variable. As simultaneous RV and photometric data of these stars are available, phase inconsistency of the data cannot explain this. In the case of the new variable, we think that the problem arises from that the used grid, interpolated to $0.05 \log g$ and $5 \mathrm{~K}$ mesh, might not be accurate enough to follow the very small-amplitude changes of the $V-I_{\mathrm{C}}$ colour $(0.015-0.020 \mathrm{mag})$. However, this cannot be the explanation for V086, which star has a similar amplitude to other overtone variables.

The amplitude of the $R_{\mathrm{ph}}$ curve of V088 is larger than the amplitude of its $R_{\mathrm{sp}}$ curve. No simultaneous photometric and spectroscopic data are available for this star. However, as no differences in the photometric amplitudes are detected according to all the available CCD photometric observations obtained in the past 25 years, the possibility that real amplitude variation would explain the differences of the $R_{\mathrm{ph}}$ and $R_{\mathrm{sp}}$ amplitudes is excluded. Both the mean RV, the proper motion (Tucholke et al. 1994), and the mean magnitude val- 
Table 10. Results of the Baade-Wesselink analysis of single-mode RR Lyrae stars in M3.

\begin{tabular}{|c|c|c|c|c|c|c|c|c|}
\hline \multirow[t]{2}{*}{ Var. } & \multirow[t]{2}{*}{ Period } & \multirow[t]{2}{*}{ Distance } & \multicolumn{6}{|c|}{$\mathrm{d}=10480 \mathrm{pc}$} \\
\hline & & & $R$ & $\Delta R^{a}$ & $T_{\text {eff }}$ & $\Delta T$ & $T_{\min 1}^{b}$ & $T_{\min 2}^{c}$ \\
\hline & days & $\mathrm{pc}$ & \multicolumn{2}{|c|}{$R_{\odot}$} & \multicolumn{4}{|c|}{ K } \\
\hline \multicolumn{9}{|c|}{ Fundamental mode } \\
\hline V009 & 0.541549 & 10600 & 5.33 & 0.84 & 6619 & 1625 & 6150 & 6425 \\
\hline V015 & 0.530088 & - & 5.34 & 0.75 & 6647 & 1735 & 6185 & 6475 \\
\hline V018 & 0.516455 & 10180 & 5.06 & 0.79 & 6681 & 1745 & 6235 & 6380 \\
\hline V019 & 0.631980 & 10700 & 5.74 & 0.66 & 6377 & 670 & 6150 & 6255 \\
\hline V021 & 0.515766 & - & 5.10 & 0.76 & 6678 & 1795 & 6205 & 6425 \\
\hline V026 & 0.597751 & 10540 & 5.74 & 0.88 & 6527 & 1435 & 6125 & 6260 \\
\hline V027 & 0.579063 & 10500 & 5.32 & 0.83 & 6631 & 1280 & 6280 & 6390 \\
\hline V036 & 0.545596 & 10180 & 5.26 & 0.84 & 6678 & 1700 & 6210 & 6380 \\
\hline V040 & 0.551539 & 10640 & 5.30 & 0.84 & 6585 & 1440 & 6185 & 6325 \\
\hline V046 & 0.613388 & 10200 & 5.71 & 0.70 & 6394 & 805 & 6120 & 6290 \\
\hline V051 & 0.583968 & 10500 & 5.49 & 0.82 & 6516 & 1235 & 6160 & 6320 \\
\hline V055 & 0.529826 & 10320 & 5.32 & 0.81 & 6595 & 1755 & 6095 & 6360 \\
\hline V060 & 0.707729 & 10280 & 6.00 & 0.85 & 6461 & 945 & 6165 & 6235 \\
\hline V064 & 0.605461 & 10420 & 5.70 & 0.82 & 6415 & 1010 & 6110 & 6255 \\
\hline V072 & 0.456079 & 10440 & 4.66 & 0.72 & 6895 & 2190 & 6325 & 6595 \\
\hline V081 & 0.529120 & 11420 & 5.26 & 0.82 & 6622 & 1640 & 6170 & 6375 \\
\hline V082 & 0.524539 & 10400 & 5.22 & 0.81 & 6647 & 1730 & 6150 & 6380 \\
\hline V083 & 0.501270 & 10600 & 5.03 & 0.79 & 6750 & 1905 & 6230 & 6495 \\
\hline V084 & 0.595729 & - & 5.72 & 0.71 & 6428 & 1080 & 6130 & 6195 \\
\hline V089 & 0.548481 & 10680 & 5.39 & 0.81 & 6622 & 1540 & 6210 & 6285 \\
\hline V090 & 0.517030 & - & 5.16 & 0.75 & 6658 & 1865 & 6165 & 6405 \\
\hline V093 & 0.602297 & 10400 & 5.76 & 0.83 & 6420 & 1105 & 6080 & 6165 \\
\hline V094 & 0.523696 & 10880 & 5.17 & 0.82 & 6660 & 1695 & 6215 & 6435 \\
\hline V096 & 0.499406 & - & 5.21 & 0.77 & 6660 & 1965 & 6135 & 6295 \\
\hline V113 & 0.513005 & - & 5.09 & 0.77 & 6721 & 1850 & 6220 & 6400 \\
\hline V115 & 0.513346 & 9680 & 5.23 & 0.75 & 6650 & 1825 & 6130 & 6365 \\
\hline V116 & 0.514809 & - & 5.11 & 0.82 & 6690 & 1760 & 6210 & 6440 \\
\hline V120 & 0.640145 & 10380 & 5.89 & 0.66 & 6326 & 710 & 6070 & 6235 \\
\hline V123 & 0.545475 & - & 5.42 & 0.85 & 6551 & 1695 & 6110 & 6270 \\
\hline V124 & 0.752440 & 10660 & 6.34 & 0.61 & 6277 & 500 & 6090 & 6170 \\
\hline V202 & 0.773575 & - & 6.38 & 0.29 & 6281 & 235 & 6180 & 6200 \\
\hline \multicolumn{9}{|c|}{ 1st overtone } \\
\hline V037 & 0.326636 & - & 4.53 & 0.22 & 7175 & 740 & 6835 & 6895 \\
\hline V056 & 0.329598 & 10180 & 4.69 & 0.22 & 7074 & 745 & 6740 & 6740 \\
\hline V086 & 0.292656 & 10460 & 4.27 & 0.19 & 7348 & 880 & 6965 & 6975 \\
\hline V088 & 0.298750 & - & 4.33 & 0.08 & 7297 & 940 & 6860 & 6870 \\
\hline V097 & 0.334930 & 10720 & 4.54 & 0.20 & 7108 & 640 & 6805 & 6815 \\
\hline V107 & 0.309031 & 10520 & 4.42 & 0.22 & 7259 & 880 & 6875 & 6880 \\
\hline \multicolumn{9}{|c|}{ 2nd overtone } \\
\hline \multicolumn{2}{|c|}{ New var. 0.249243} & - & 3.85 & 0.02 & 7755 & 90 & 7710 & 7710 \\
\hline & Median: & 10480 & & & & & & \\
\hline & Mean: & 10480 & & & & & & \\
\hline & S.dev.: & 310 & & & & & & \\
\hline
\end{tabular}

${ }^{a} \Delta R$ is the peak to peak amplitude of the spectroscopic radius curve.

${ }^{b} T_{\min 1}$ is the absolute minimum value of the temperature variation.

${ }^{c} T_{\min 2}$ is the temperature value at the phase of light minimum.

ues of V088 are consistent with its cluster membership, so it is unlikely that its distance would differ significantly from the accepted 10480 pc value. Most probably, some defect of the Hydra spectra of this star results in an anomalously small amplitude of its $R_{\mathrm{sp}}$ curve.

The $4-8$ th columns of Table 10 list the mean radius, the amplitude of the radius variation according to the spectroscopic data, the mean and the amplitude of $T_{\text {eff }}$, and the minimum temperature values derived twofold. $T_{\min 1}$ and $T_{\min 2}$ correspond to the absolute minimum temperature value occurring in the $0.45-0.60$ pulsation-phase interval of RRab stars, and the temperature at light minimum, at phase $0.8-0.9$, respectively. 


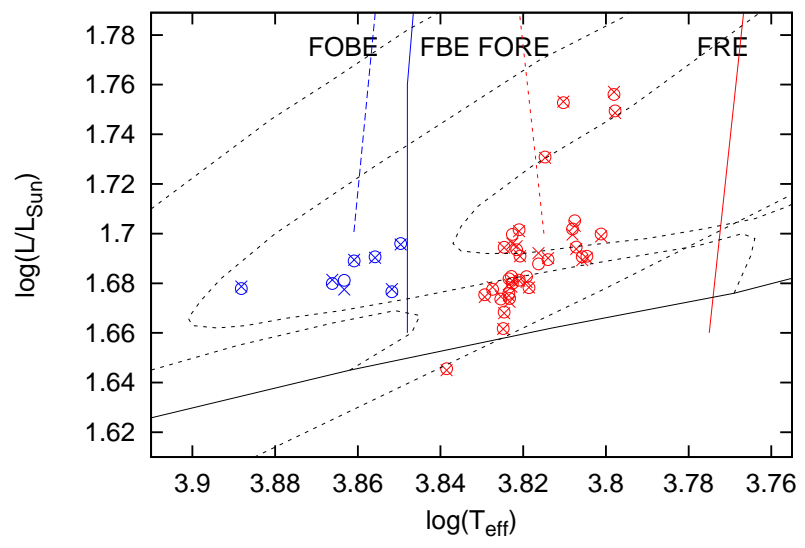

Figure 10. The instability strip of M3, according to the data of the RR Lyrae stars that are the subject of the Baade-Wesselink analysis, is shown. Overtone and fundamental-mode variables are shown by blue and red symbols, respectively. The luminosities, calculated using the two luminosity formulae, are shown by circles and crosses. The blue and red edges of the first-overtone and the fundamental-mode instability strips (FOBE, FBE, FORE and FRE, Marconi et al. 2015) are also indicated by red and blue lines. The hottest star beyond the first-overtone blue edge is the new variable, which is probably a second-overtone RRL. The zero-age horizontal branch according to the $\alpha$ enhanced $[\mathrm{Fe} / \mathrm{H}]=-1.48$ models of Dorman $(1992)$ and the $[\mathrm{Fe} / \mathrm{H}]=-1.5$ and $[\alpha / \mathrm{Fe}]=0.4$ models of Dotter et al. (2007) are drawn by continuous and dotted black lines, respectively. The 0.62, 0.64 and $0.66 M_{\odot}$ horizontal-branch evolutionary tracks of the Dorman models are also shown.

\section{COMPARISON WITH RESULTS OF THEORETICAL AND EMPIRICAL STUDIES}

\subsection{The instability strip}

The instability strip occupied by the RRL stars of M3 is shown in Figure 10, in comparison with model results of its boundaries according to the $\mathrm{Z}=0.001$ models of Marconi et al. (2015). The luminosities of the stars are calculated twofold; the $L / L_{\odot}=R / R_{\odot}{ }^{2} \times T / T_{\odot}{ }^{4}$, and the $-2.5 \log L / L_{\odot}=V_{0}+B C-5 * \log d+5-M_{\mathrm{Bol}, \odot}$ formulae are applied. Only stars that are the subject of the BW analysis are shown. The $T_{\text {eff }}, R$ and B.C. are determined in the course of the BW analysis, and the distance is taken to be $10480 \mathrm{pc}$.

The theoretical red boundary of both the fundamental and of the first overtone mode is at about $300 \mathrm{~K}$ cooler temperature than defined by the sample of RRL stars shown. The hottest star observed, the new variable $\left(T_{\text {eff }}=7755 \mathrm{~K}\right)$, lays at a $500 \mathrm{~K}$ hotter temperature than the blue edge of the first-overtone instability region is, supporting that this star is a second-overtone RRL.

For comparison, the location of the zero-age horizontal branch according to appropriate composition stellar evolution models of Dorman (1992) and Dotter et al. (2007) are also indicated in Fig. 10.

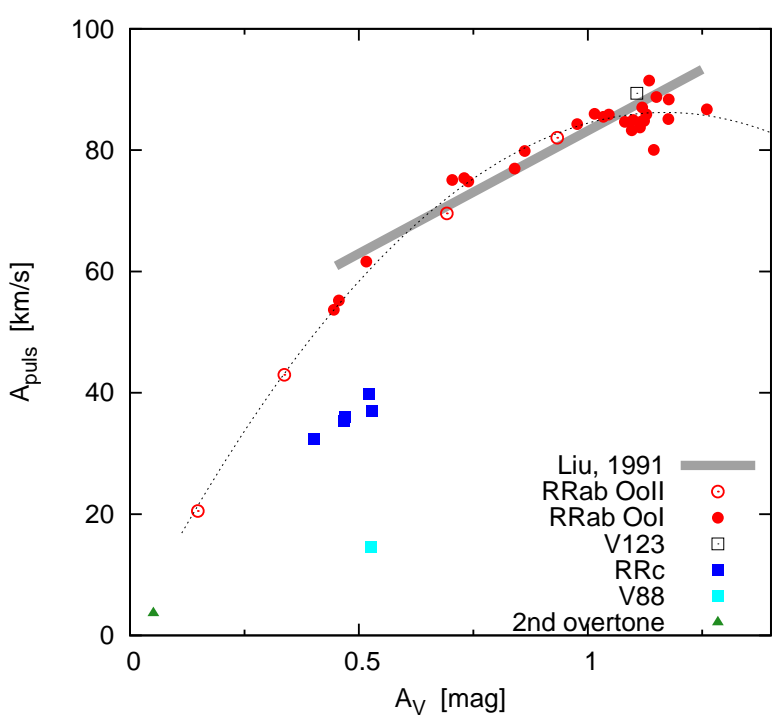

Figure 11. Pulsation amplitude $\left(p * A_{\mathrm{RV}}\right)$ versus the full amplitude of the $V$ light curve of RR Lyrae stars in M3 is compared with the $A_{\text {puls }}-A_{V}$ relation determined by Liu (1991) (gray line) for Galactic field RRab stars.

\section{2 $A_{\text {puls }}-A_{V}$ relation of RRL stars}

Based on photometric and spectroscopic data, Liu (1991) derived a linear relation between the pulsation amplitude $\left(A_{\mathrm{RV}}\right.$ puls $=p A_{\mathrm{RV}} ; p$ :projection factor $)$ and the $V$ amplitude of stable-light-curve RRab stars. The $A_{\mathrm{RV}}-A_{V}$ relation of a Blazhko star in different phases of the modulation seems, however, to be star by star different and steeper than that of stable RRab stars (Jurcsik et al. 2002).

The $A_{\text {puls }}-A_{V}$ relation determined by Liu (1991) in comparison with the M3 results is shown in Fig. 11. Liu's relation gives a very good linear approximation of the M3 data, however, covering a larger amplitude (period) range, the relation is clearly nonlinear. The following formula

$A_{\text {puls }}=145.46 A_{V}-62.22 A^{2}{ }_{V}+1.21$

fits the data of stable RRab stars in M3 with $2.2 \mathrm{~km} \mathrm{~s}^{-1} \mathrm{rms}$ scatter. It is important to note, that this relation is uniquely valid for OoI and OoII variables.

\subsection{Period-radius relation}

The period dependence of the obtained mean radius values of the variables are compared with model results in Fig. 12. The period-radius (PR) relations defined by the fundamental and the first-overtone RRL stars and the theoretical relations for $\mathrm{z}=0.001$ metallicity value (eq. 7 . and 8 . in Marconi et al. 2015) are shown in this figure. The fitted relations have the form of

$\log \left(R / R_{\odot}\right)=0.872( \pm 0.006)+0.555( \pm 0.025) \log P_{\mathrm{FU}}$

$\log \left(R / R_{\odot}\right)=0.94( \pm 0.06)+0.57( \pm 0.12) \log P_{\mathrm{FO}}$

for the fundamental $(\mathrm{FU})$ and the first overtone (FO) variables, respectively.

The agreement between the theoretical and the observed PR relations is excellent; both the slopes and the 


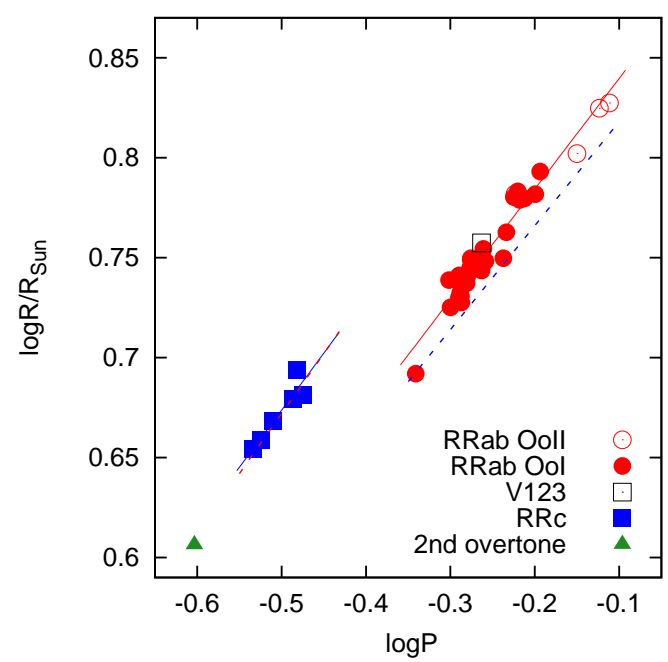

Figure 12. Period - radius relations of RR Lyrae stars in M3. The linear fits to the RRab and RRc data (continuous lines) and the $P-R$ relations derived by Marconi et al. (2015) (dashed lines) are also shown. The two lines are practically identical for RRab stars and the offset between the empirical and theoretical relations of RRc stars is only about 0.015 in $\log R$.

zero points (if corrected for the metallicity dependence) of the relations are the same within the $1 \sigma$ limit for both the RRab and the RRc stars. Although the theoretical $\log R$ values seem to be larger by 0.015 than the radii determined from the BW analysis, this difference remains within the uncertainty limit, moreover, such a difference can arise e.g., from a minor, $\sim 0.10$ offset of the $\log g$ values accepted for the overtone variables.

\subsection{Minimum colour and temperature values}

Fig. 13 plots the period dependence of the mean and the $\min 1$ and $\min 2$ values of the dereddened $(V-I)_{0}$ and $T_{\text {eff }}$ (see Table 10) of non-Blazhko and non-double-mode RRL stars in M3. The min1 corresponds to the red (cold) extreme of the colour (temperature) again, while min2 denotes their values at light minimum.

While the mean colours and the temperature of RRL stars depend on the period of the pulsation strongly, the colours and the temperature at light minimum have been supposed to be nearly the same at any period value especially for fundamental-mode variables. Guldenschuh et al. (2005) derived a $(V-I)_{0, \min }-[\mathrm{Fe} / \mathrm{H}]$ relation using the average value of the $(V-I)_{0}$ over the $0.5-0.8$ pulsationphase range for Galactic field RRL stars. This relation yields $(V-I)_{0, \min }=0.58 \mathrm{mag}$ value for the metallicity of M3. The mean of the $(V-I)_{0, \min 1}$ values of the RRab stars in M3 is 0.583 , which is in excellent agreement with this result.

Studying the period-colour relations of RRL stars using the OGLE III data (Soszyński et al. 2009, 2010) of the Large and Small Magellanic Clouds (MCs), Bhardwaj et al. (2014) found that the slope of the $(V-I)_{0}$ versus $\log P$ relation for RRab and RRc stars are 0.093/0.055 and 0.604/0.472 in the LMC/SMC, respectively. $(V-I)_{0}$ corresponds the min2 data here, measured at the phase of the light minimum. The linear fits to the RRab data shown in the left-hand panel of Fig. 13 have, however, significantly larger, 0.502 and 1.26 slopes for the min2 data for the fundamental and the overtone variables, respectively. These are 2-10 times larger than the corresponding values determined for the data of the MCs. Although the period dependence of the min1 data of RRab stars is significantly smaller than for min2, its slope is still not negligible for the $(V-I)_{0, \min 1}$ data. The form of the linear fits to the min1 data of the $(V-I)_{0}$ and the $T_{\text {eff }}$ data of RRab stars shown in Fig. 13 are the followings:

$$
\begin{aligned}
(V-I)_{0, \min 1} & =0.65( \pm 0.02)+0.26( \pm 0.06) \log P_{\mathrm{FU}} \\
T_{\mathrm{eff}, \min 1} & =3.781( \pm 0.003)+0.037( \pm 0.013) \log P_{\mathrm{FU}}
\end{aligned}
$$

Concerning the overtone variables, the period dependences of the $(V-I)_{0}$ values using min 1 and min2 data are practically the same; their steepness is 1.26 .

Clearly, the relative constancy of the minimum temperature and the $(V-I)_{0}$ colour of RRL stars is valid only for the fundamental mode and for the absolute minimum values (min1), while the mean and the minimum-light colors are strongly period dependent if a homogeneous sample of variables, like in M3, is investigated.

The discrepancy between the detected period dependencies of the minimum colours in M3 and in the MCs arises, most probably, from that the scarceness of the $V$ light curves and the larger uncertainties of the photometric data hinder to determine the $(V-I)_{0, \text { min }}$ values in the MCs accurately enough.

\section{$8 \quad \mathrm{~V} 123$}

The peculiar light-curve shape of V123 was discussed in detail in Jurcsik et al. (2013). Based on its brightness, colours and amplitudes, V123 is similar to RRab stars of the same period in M3, but its light-curve has a smaller bump and a longer and less steep rising branch than other, stable-lightcurve RRab stars have. Although the light curve resembles to the light curves of some Blazhko stars, no clear evidence of the Blazhko effect has been detected; only very smallamplitude stochastic variation around maximum brightness is evident.

In spite of that the position of V123 was a bit offset in the Hectochelle@MMT observations and the spectra have low $\mathrm{S} / \mathrm{N}$, reasonable RV data of these spectra could also be obtained. The scatter of this RV curve is larger than for the other RRL stars, however, this is the consequence of the low quality of the spectra, and does not indicate real RV variations.

In order to find an answer to the peculiarities of V123, a complete BW analysis of this star is also performed. The observed and fitted $V$ and RV curves and the matching of the $R_{\mathrm{sp}}$ and $R_{\text {phot }}$ radius curves using different phase intervals for the fit are shown in Fig. 14. The phase intervals involved in the fitting process and the derived distances are given in the right-hand side of the panel for the BW solutions shown. If the phase interval between 0.8 and 1.1 is omitted, the distance derived for V123 is 10440 pc, in perfect agreement with the mean distance value obtained for M3. If smaller or no phase interval is skipped, then the distance become 

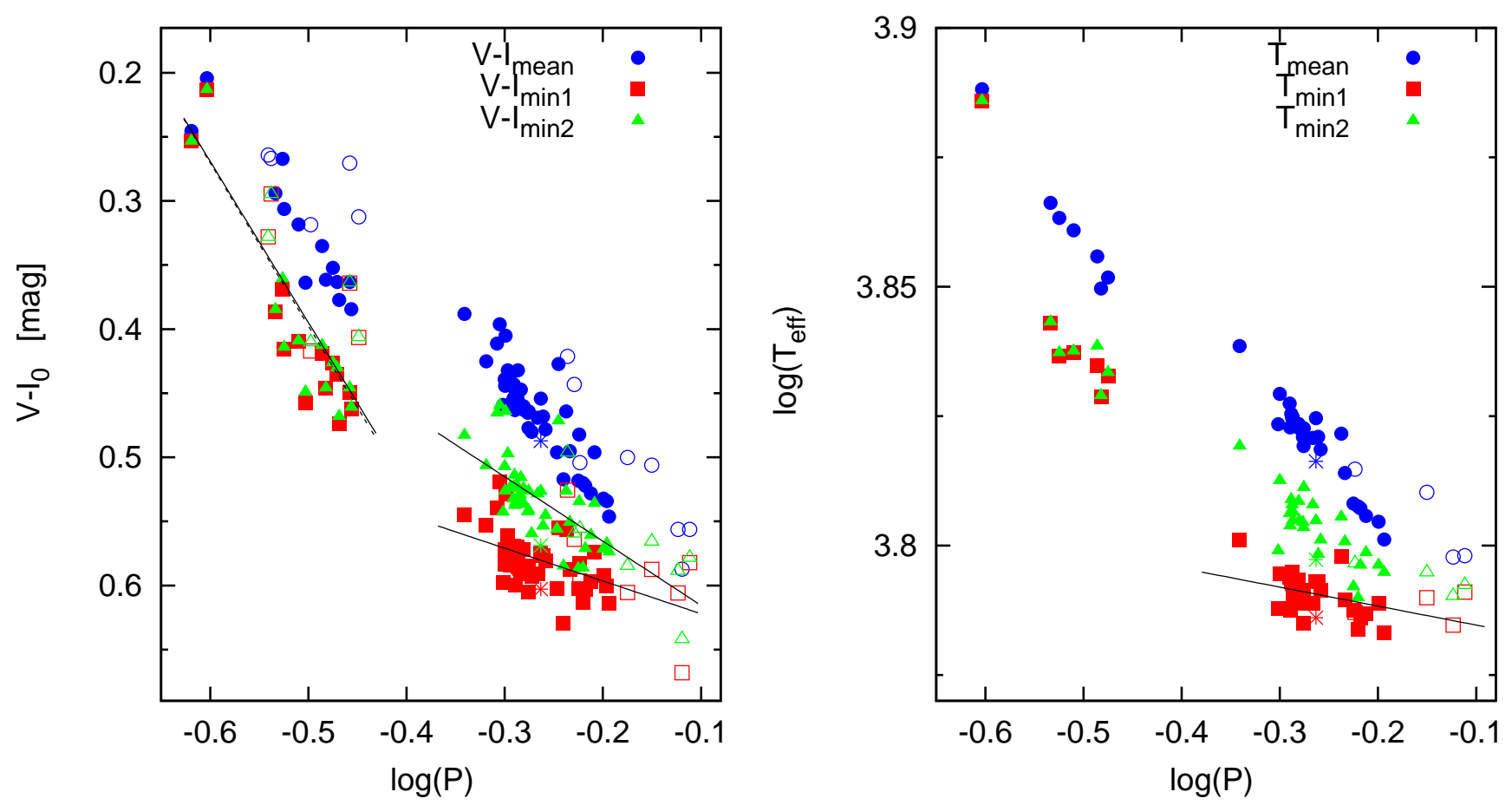

Figure 13. Mean and extrema of the $(V-I)_{0}$ (maximum) and effective temperature (minimum) data of RR Lyrae stars. The min1 and min2 denote the red/cold extrema and the corresponding values at light minimum, respectively. The open symbols denote OoII stars. All the stable-mode RR Lyrae stars with accurate $V$ and $I_{\mathrm{C}}$ photometric data are shown in the left-hand panel, and those which have both photometric and radial-velocity data are plotted in the right-hand panel. Note, that the values of the both the min1 and the min2 data are period dependent, and the period dependence of the min2 data of RRab stars is significantly larger than that of the min1 data. V123 is shown by star symbols.

larger, and a systematic difference between the radius curves emerges in the $0.6-0.8$ phase interval. In these solutions, the $R_{\mathrm{sp}}$ is larger than $R_{\text {phot }}$ all along the $0.6-0.8$ phases, which is not the case for any of the other RRab stars analysed as it can bee seen in Fig. 8.

Therefore, the BW analysis of V123 seems to strengthen the conclusion that the star is indeed a cluster member. The $R_{\mathrm{sp}}$ and $R_{\text {phot }}$ curves of V123 using the adopted 10480 pc distance of the cluster is shown in the right-hand panel of Fig. 8. The matching of the two radius curves is similarly good as for other RRab stars between phases 0.15 and 0.80 , however, the discrepancy between the two curves is the largest in the $0.80-1.15$ phase interval in the whole sample of the stars analysed. Typically, $R_{\text {phot }}$ is $0.1-0.2 R_{\odot}$ larger than $R_{\mathrm{sp}}$ between phases 0.9 and 1.1 for the shorter period, large-amplitude RRab stars, i.e. in those stars which develop large atmospheric shocks. In the case of V123, the discrepancy of the radius curves affects a longer phase interval and its amplitude is also larger $\left(0.2-0.3 R_{\odot}\right)$ than in the other stars.

The large discrepancy of the radius curves around the rising branch of the light curve indicates strong anomalies of the atmosphere in these phases in line with that the biggest discrepancy in the shape of the light curve appears also in this phase interval. Most probably, an anomalous, strong shock, appearing at a peculiar phase, is behind the anomalies of V123.
The positions of V123 are shown by special symbols in the different figures of the present paper. It was already shown in Jurcsik et al. (2013) that the $R_{k 1}(k>2)$ Fourier amplitude ratios and the higher-order phase differences $\varphi_{k 1}$ $(k>5)$ of V123 are anomalously small, as it is also documented in Fig. 6. This is in contrast with the behaviour of the Fourier parameters of the RV curve. Only the $A_{5}, A_{6}$, $A_{7}$ amplitudes and the $R_{51}, R_{61}$ and $R_{71}$ amplitude ratios are smaller than normal, but no significant anomaly of the phase differences of the RV curve is evident. This is in line with that the shape of the RV curve does not look to be so peculiar as the shape of the light curve is (see Fig. 14).

The $A_{\text {puls }}-A_{V}$ relation, the radius and the minimum colours and temperatures of V123 are normal, as documented in Fig. 11, 12 and 13. It seems that, with the exception of its light-curve shape, all the properties of V123 are similar to the properties of a normal RRab star.

\section{SUMMARY}

Radial velocity and photometric data of a large set of RRL variables in M3 are published. This is the first extended RV survey of RRL stars in a GC. Moreover, this is the largest set of RV curves of RRL stars ever obtained.

The main results derived from these data are as follows.

- $B V I_{\mathrm{C}}$ magnitude of 111 and instrumental $B$-band flux 


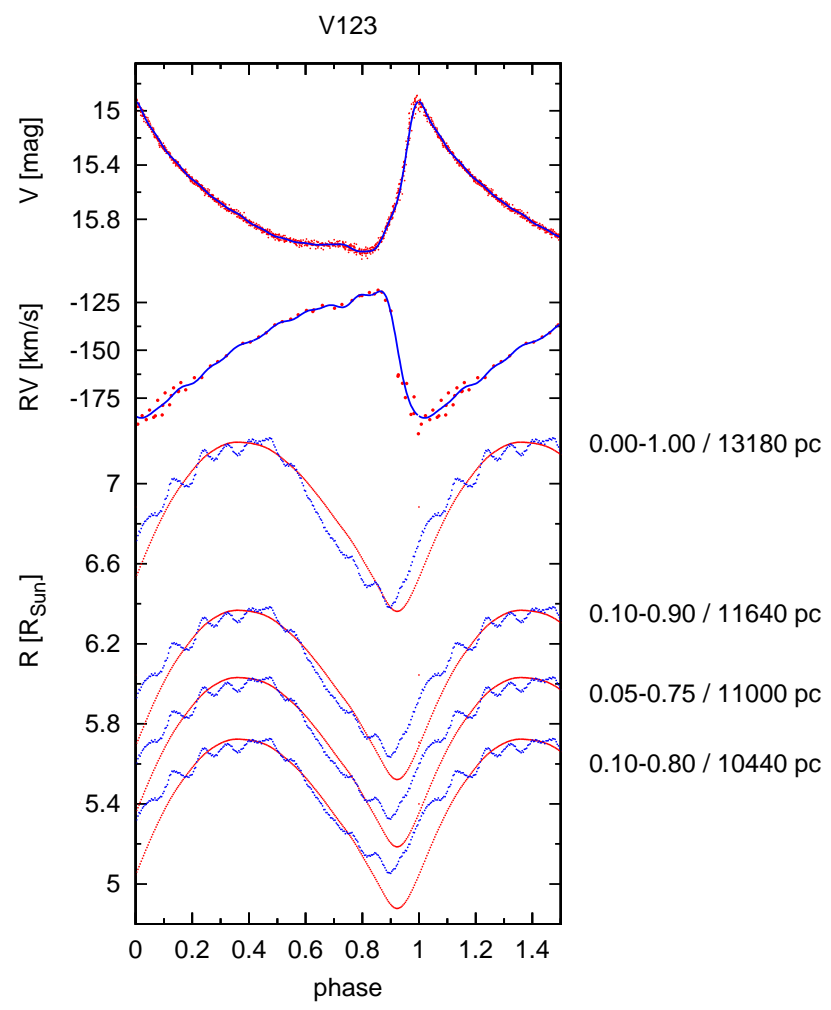

Figure 14. The observed and the fitted $V$ and radial-velocity curves and the $R_{\mathrm{sp}}$ and $R_{\mathrm{phot}}$ variations according to different Baade-Wesselink solutions are shown for V123. Different distances are derived if different phase intervals of the $R_{\mathrm{sp}}$ and $R_{\mathrm{phot}}$ curves are concerned in the fitting process. The phase interval of the fit, and the derived distances are given in right side of the panel for some solutions.

time series of 64 RRab stars are presented. The light curves of 47 percent of the variables show the Blazhko effect.

- The mean value of the $\gamma$-velocities of $79 \mathrm{RRL}$ stars is $-146.8 \mathrm{~km} \mathrm{~s}^{-1}$ with $4.52 \mathrm{~km} \mathrm{~s}^{-1}$ standard deviation. The velocity dispersion of RRL stars at different projected radial distances $(r<12$ arcmin) are slightly but systematically smaller than the velocity dispersion of giants, however, the differences are not statistically significant.

- Difference of about $1-2 \mathrm{~km} \mathrm{~s}^{-1}$ between the mean RVs of Blazhko stars in different phases of the modulation are detected. This is probably a consequence of the dynamics of the atmosphere and/or the reduction process, and does not reflect real changes of the $\gamma$-velocities.

- The $\Phi_{21}{ }^{R V}$ phase differences of the RV curves of RRab stars seem to be uniformly constant. Comparing the $\Phi_{21}{ }^{R V}$ values of M3 and Galactic field stars, no period or metallicity dependence, larger than the uncertainty would indicate, has been found.

- The mean value of the BW distances of 26 single-mode RRL stars is $10480 \pm 210 \mathrm{pc}$, which is in good agreement with literature data.

- The $A_{\text {puls }}-A_{V}$ relation of RRab stars is quadratic, and OoI and OoII stars obey the same relation.

- The period - radius relation defined by the results of the
BW analysis of 6 RRc and 31 RRab stars are in very good agreement with theoretical model results (Marconi et al. 2015).

- The $(V-I)_{0}$ colour and the temperature at the phase of minimum light is found to show a clear period dependence; the differences between the shortest and longest period RRab stars are about $0.1 \mathrm{mag}$ and $500 \mathrm{~K}$. The period dependence of the absolute minimum value of the temperature (the absolute maximum values of the colours), which occur at pulsation phase between 0.45 and 0.60 , is less significant. The mean values of the extrema of the $(V-I)_{0}$ and temperature data of non-Blazhko RRab stars in M3 are $0.583 \pm 0.025 \mathrm{mag}$ and $6170 \pm 60 \mathrm{~K}$, respectively.

A detailed analysis of the photometry and RV data of Blazhko stars is going to be published in forthcoming papers.

\section{ACKNOWLEDGMENTS}

The constructive comments of the referee, Antonio Sollima, helped to inprove the paper. GH acknowledges support by the Ministry for the Economy, Development, and Tourism's Programa Iniciativa Científica Milenio through grant IC210009, awarded to the Millennium Institute of Astrophysics; by Proyecto Basal PFB-06/2007; by Fondecyt grant 1141141; and by CONICYT-PCHA/Doctorado Nacional grant 2014-63140099. ÁS and KV were supported by the János Bolyai Research Scholarship of the HAS and PS by PECS-98073. KK gratefully acknowledges the support of a Marie Curie IOF under FP7 (PIOF-255267 SAS-RRL). CAP gratefully acknowledges support from the Daniel Kirkwood endowment at Indiana University. NJ acknowledges the NKFIH K-115709 grant, MA the grant of the MTA CSFK Lendület Disk Research Group, AP the OTKA K113117 and LP2012-31 grants. KS has been supported by the Hungarian OTKA grant K-104607.

\section{REFERENCES}

Alard C. 2000, A\&A Suppl., 144, 363

Benkő J. M., Bakos G. Á. Nuspl, J. 2006, MNRAS, 372, 1657

Bhardwaj A., Kanbur S. M., Singh H. P., Ngeow C.-C. 2014, MNRAS, 445, 2655

Cacciari C., Clementini G., Fernley J. A. 1992, ApJ, 396, 219

Cacciari C., Corwin T.M., Carney B.W. 2005, AJ, 129, 267

Carney B. W., Storm J., Jones R. V. 1992, ApJ, 386, 663

Carney B. W. 1996, PASP, 108, 900

Cassisi S., De Santis R., Piersimoni A. M. 2001, MNRAS, 326, 342

Castellani M., Castellani V., Cassisi S. 2005 A\&A, 437, 1017

Castelli F. Kurucz R. L. 2003, in Modelling of Stellar Atmospheres, ed. N. Piskunov, W.-W. Weiss and D.-F. Gray, IAU Symp., 210, 20

Catelan, M. 2004 ApJ, 600, 409

Chadid, M., Preston G. W. 2013, MNRAS, 434, 552

Cohen J. G. 1992, ApJ, 400, 528

Cohen J. G., Meléndez J. 2005, AJ, 129, 303

Clementini G., Corwin T. M., Carney B. W., Sumerel A.N. 2004, AJ, 127, 938

Clementini G., Cacciari C., Lindgren H. 1990, A\&A Suppl., 85, 865

Corwin B. W., Carney B. W. 2001, AJ, 122, 3183

Dorman B., 1992, ApJ Suppl., 81, 221 
Dotter A., Chaboyer B., Jevremović D., Baron E., Ferguson J. W., Sarajedini A., Anderson J. 2007, AJ, 134, 376

Feuchtinger M. U. 1999, A\&A, 351, 103

Fernley J., 1994, A\&A, 284, 16

Gautschy A. 1987, Vistas in Astronomy, 30, 197

Gillet, D., \& Fokin, A. B. 2014, A\&A, 565, 73

Givens R. A., Pilachowski C. A. 2016, PASP, 128, 4203

Guldenschuh K. A. et al. 2005, PASP, 117, 721

Gunn J. E., Griffin R. F. 1979, AJ, 84, 752

Harris W. E. 1996 (2010 edition), AJ, 112, 1487

Hartman J. D., Kaluzny J., Szentgyörgyi A., Stanek K. Z. 2005, AJ, 129, 1596

Hernandez X., Jimènez M. A. 2012, ApJ, 750, 9

Johnson C. I., Kraft R. P., Pilachowski C. A., Sneden C., Ivans I. I., Benman G. 2005, PASP, 117, 1308

Jones R. V., Carney B. W., Storm J., Latham D. W. 1992, ApJ, 386,646

Jurcsik J., 1998, A\&A, 333, 571

Jurcsik J., Kovács, G. 1996, A\&A, 312, 111

Jurcsik J., Benkő, J., Szeidl, B. 2002, A\&A, 390, 133

Jurcsik J., Hajdu G., Szeidl B. et al. 2012, MNRAS, 419, 2173

Jurcsik J. et al 2013, ApJ Letters, 776, L1

Jurcsik J. et al. 2015, ApJ Suppl. 219, 25

Jurcsik J., Smitola P. 2016, Communications from the Konkoly Observatory, Vol. 105, p. 167

Kaluzny J., Hilditch R. W., Clement C., Rucinski S. M. 1998, MNRAS, 296, 347

Kamann, S. et al., 2014, A\&A, 566, A58

Kimmig B., Seth A., Ivans I. I., Strader J., Caldwell N., Anderton T., Gregersen D. 2015, AJ, 149, 53

King I.R. 1966, AJ 71,64

Kolláth Z. 1990, Occ. Techn. Notes Konkoly Obs., No. 1, http://www.konkoly.hu/staff/kollath/mufran.html

Kovács G. 2003, MNRAS, 342, L58

Kovács G., Walker A. R. 2001, A\&A, 371, 579

Liu T. 1991, PASP, 103, 205

Liu T., Janes K. A. 1990, ApJ, 354, 273

Liu T., Janes K. A., 1990, ApJ, 360, 561

Marconi M et al. 2015, ApJ, 808, 50

Marconi M., Caputo F., Di Criscienzo M., Castellani M. 2003, ApJ, 596, 299

Marconi M., Degl'Innocenti S. 2007, A\&A, 474, 557

Marengo M., Karovska M., Sasselov D. 2004, ApJ, 603, 285

Moskalik P., Krzyt T., Gorynya N. A., Samus N. N. 2000, in The Impact of Large-Scale Surveys on Pulsating Star Research, ed. L. Szabados and D. Kurtz, ASPC, 203, 233

Moskalik P. 2014, in Precision Asteroseismology, ed., J.A. Guzik, W.J. Chaplin, G. Handler \& A. Pigulski, Proceedings IAU Symposium No. 301, 249

Nardetto N., Fokin A., Mourard D., Mathias Ph., Kervella P., Bersier D. 2004, A\&A, 428, 131

Navin C. A., Martell S. L., Zucker D. B. 2016, ApJ, 829, 123

Pilachowski C. A., Sneden C., Kraft R.P., Harmer D., Willmarth D. 2000, AJ, 119, 2895

Pont F., Kienzle F., Gieren W., Fouqué P. 2001, A\&A, 376, 892

Preston G. W. 2011, AJ, 141, 6

Samus N. N., Kazarovets E. V., Pastukhova E. N., Tsvetkova T. M., Durlevich O. V. 2009, PASP, 121, 1378

Sandstrom K., Pilachowski C. A., Saha A. 2001, AJ, 122, 3212

Simon N. R., Teays T. J. 1982, ApJ, 261, 586

Skillen I., Jameson R. F., Fernley J. A., Lynas-Gray A. E., Longmore A. J. 1989, MNRAS, 241, 281

Skillen I., Fernley J. A., Stobie R. S., Jameson R. F. 1993, MNRAS, 265, 301

Smart R. L., Nicastro L. 2014, A\&A, 570, 87

Smolinski J.P., Martell S.L., Beers T.C., Lee Y. S. 2011, AJ, 142, 126
Sneden C., Kraft R. P., Guhathakurta P., Peterson R.C., Fulbright J. P. 2004, AJ, 127, 2162

Soderberg A. M., Pilachowski C. A., Barden S. C., Willmarth D., Sneden C. 1999, PASP, 111, 1233

Sódor Á. 2012 Occ. Techn. Notes Konkoly Obs., No. 1, http://www.konkoly.hu/staff/sodor/lcfit.html

Sollim, A., Cacciari C., Valenti E. 2006, MNRAS, 372, 1675

Soszyński I. et al. 2009, AcA, 59, 1

Soszyński I. et al. 2010, AcA, 60, 165

Stetson, P. B. 2000, PASP, 112, 925

Storm J., Carney B. W., Latham D. W. 1994, A\&A, 290, 443

Storm J., Carney B. W., Nordström B., Andersen J., Latham D. 1994, in Hot Stars in the Galactic Halo, ed. Saul J. Adelman and Arthur R. Upgren. Cambridge: Cambridge University Press, p.298

Storm J., Nordstrom B., Carney B. W., Anderson J. 1994, A\&A, 291,121

Szeidl B., 1988, in Multimode Stellar Pulsation, ed. G. Kovács, L. Szabados \& B. Szeidl (Budapest), p51

Szentgyörgyi A., Fürész G., Cheimets P. et al. 2011, PASP, 123, 1188

Tonry J., Davis M. 1979, AJ, 84, 1511

Torres G. 2010, AJ, 140, 1158

Tucholke H.-J., Scholz R.-D., Brosche P. 1994, A\&AS, 104, 161

VandenBerg D. A., Denissenkov P. A., Catelan M. 2016, ApJ, 827,2

VandenBerg D. A., Stetson P. B., Brown T. M. 2015, ApJ, 805, 103 